Original Research Paper

\title{
The SEIR Dynamical Transmission Model of Dengue Disease with and Without the Vertical Transmission of the Virus
}

\author{
${ }^{1}$ Pratchaya Chanprasopchai, ${ }^{2}$ I. Ming Tang and ${ }^{1}$ Puntani Pongsumpun \\ ${ }^{I}$ Department of Mathematics, Faculty of Science, King Mongkut's Institute of Technology Ladkrabang, \\ Chalongkrung Road, Ladkrabang, Bangkok 10520, Thailand \\ ${ }^{2}$ Computational and Applied Science for Smart Innovation Cluster (CLASSIC), \\ Faculty of Science, King Mongkut's University of Technology Thonburi, Bangkok 10140, Thailand
}

\author{
Article history \\ Received: 18-10-2017 \\ Revised: 05-12-2017 \\ Accepted: 27-12-2017 \\ Corresponding Author: \\ Puntani Pongsumpun \\ Department of Mathematics, \\ Faculty of Science, King \\ Mongkut's Institute of \\ Technology Ladkrabang, \\ Chalongkrung road, \\ Ladkrabang, Bangkok 10520, \\ Thailand \\ Email:kppuntan@kmitl.ac.th
}

\begin{abstract}
The transmission of dengue disease when there is a possibility of Vertical Transmission (VT) is studied using mathematical modeling. In the normal case, the mosquito is infected by the dengue virus when it bites an infectious human being. Evidence is gathering that the mosquitoes can also be infected through sexual contact with infected male mosquito. To see the possible consequence of having this addition mode of transmission, a SEIR, Susceptible-Exposed-Infected-Recovery, model is constructed. The Routh Hurwitz criteria are applied to the model in order to establish the stability of the infection. It is seen that the model without the VT model has 2 equilibrium points, a disease free equilibrium point and an endemic equilibrium point, while the model with the VT has only an endemic equilibrium point. The numerical solutions of differential equations of the model without the VT mode exhibit dynamical behaviors that converges to the disease free equilibrium state when basic reproduction time $R_{0}$ is less than 1 and converges to endemic equilibrium state when $R_{0}>1$. The trajectories of the numerical solutions for all possibilities (with and without VT mode) projected onto various 2D planes and 3D spaces are presented.
\end{abstract}

Keywords: Dengue Disease, Vertical Transmission Infection Mode, SEIR Model, Disease Free and Endemic Equilibrium State, Routh - Hurwitz Criteria, Basic Reproductive Number

\section{Introduction}

Dengue disease is found in the tropical and subtropical areas around the world such as in South-East Asia, the Western Pacific, America, African, Eastern Mediterranean and others (WHO, 2011). Dengue infection is estimated to infect 50-100 million populations with almost half of world' population living in dengue endemic countries (WHO, 2012). Dengue virus has four serotypes; i.e., DEN1, DEN2, DEN3 and DEN4 and cannot transmit from human to human directly. Dengue virus is transmitted to human by the bite of infected Aedes mosquitoes. It means that human is the main host of the dengue virus and mosquito are the vectors of the transmission. The mosquitoes can be found around houses and are infected when they bite an infectious human. This leads to the dengue virus moving within and between communities. The way to control dengue disease is focused on the spreading of the mosquitoes.
In Thailand, dengue has been reported from all regions including the Bangkok metropolitan area. The reported cases and death cases from 2003 to 2015 are shown in Fig. 1. In 2015, the reported cases and deaths cases were 144,952 and 148 respectively (Bureau of Epidemiology, 2015). The historical reported data is indicated that dengue disease has potential to spread quickly with the country experiencing large epidemic in both reported cases and death. There is a high risk potential for the spread of the dengue disease when there is a high rate of contact between the host and vector in a large population of human and mosquito (as in the Bangkok metropolitan). Rainy season is suitable for mosquito to lay their eggs and Fig. 2 shows that there is high number of reported cases during the rainy season. Female mosquitoes will become the vector for the disease when they feed on the blood of infectious human. As the results, the mosquito will be able to transmit the virus to an uninfected human when she bites him. 


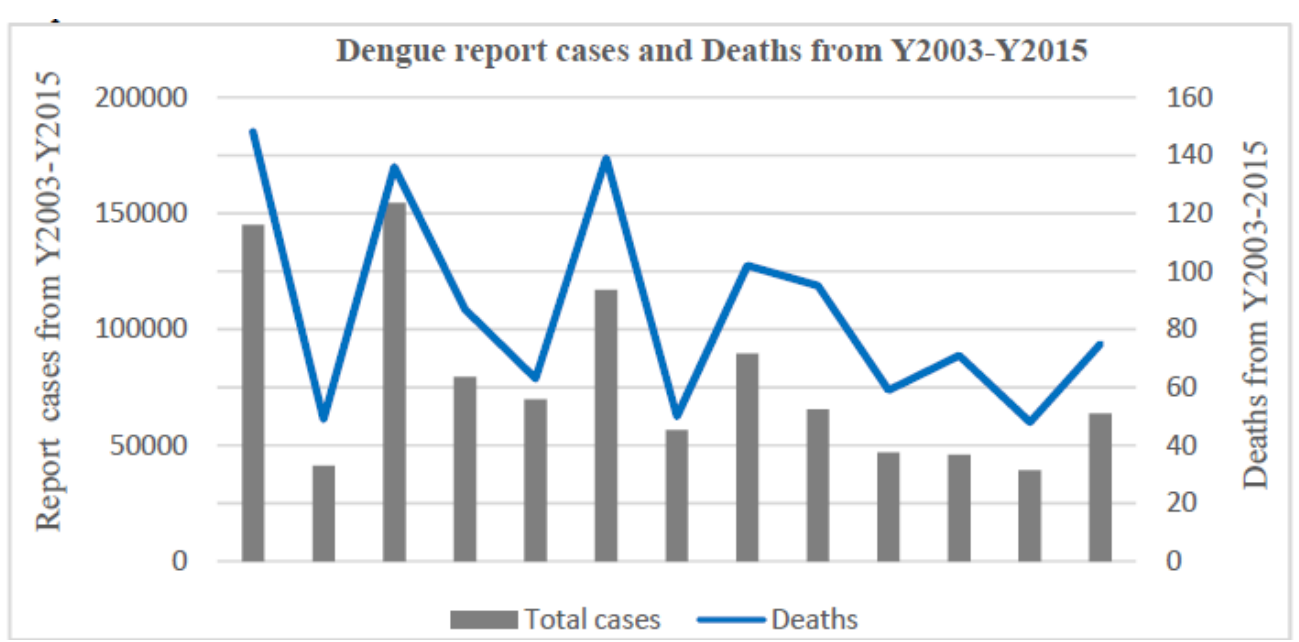

Fig. 1: Thailand dengue reported historical data from 2003 to 2015

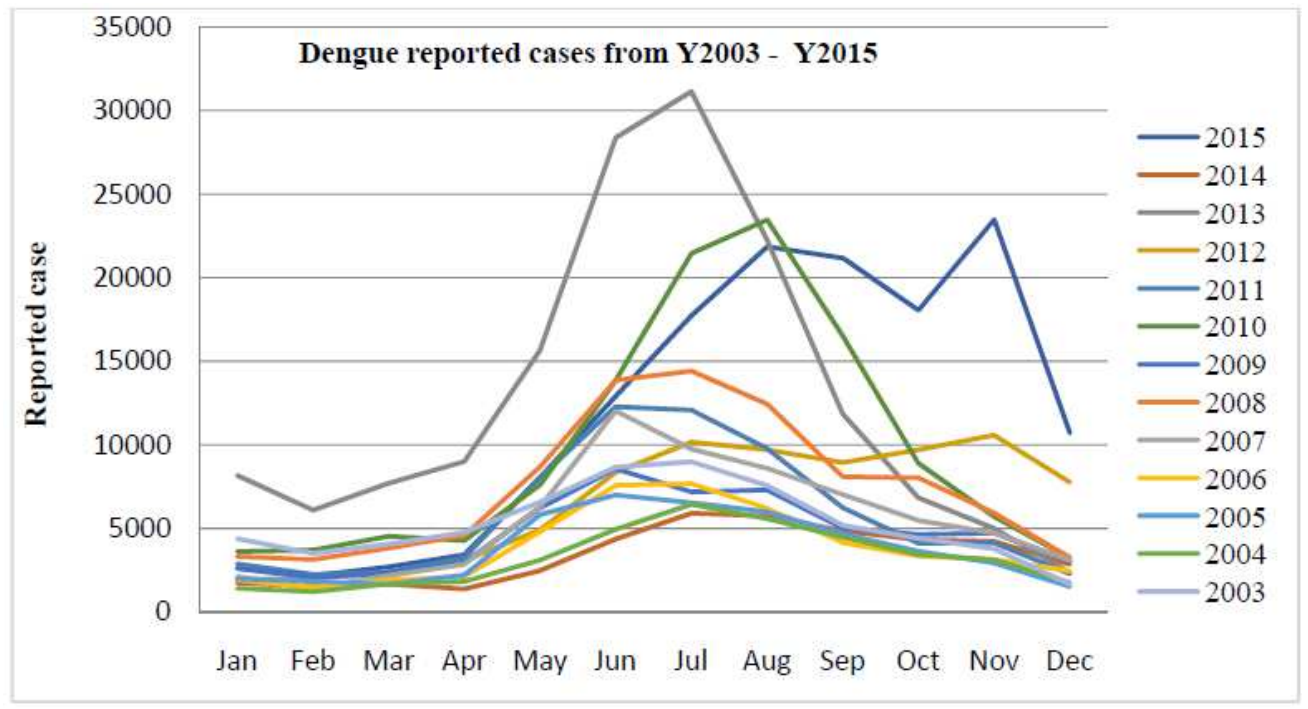

Fig. 2: Thailand dengue reported cases from 2003 to 2015 (month by month)

A model of the dengue epidemic is necessary to better understand the mechanism and behavior in order to analyze the spread and control the spread of the disease. Mathematical models are often developed to describe the transmission of dengue disease. Esteva and Vargas (1999) proposed a model for the transmission of dengue fever where the human population is constant but mosquito population varies. They provided a global analysis to establish the global stability of the endemic equilibrium. Naowarat et al. (2011) proposed a dynamical model to determine the human susceptibility to dengue fever. The standard method was used to analyze the dynamic of dengue disease system. Pongsumpun and co-workers proposed mathematical models for dengue disease which took into account additional features of the disease. The dynamic transmission with the effect of extrinsic incubation period was included. An standard dynamic analysis was applied to a modified Susceptible-Infected-Recovered (SIR) model included an annual variation in the length of the extrinsic incubation period in the mosquito (Pongsumpun, 2006a). The dynamic transmission behaviors of dengue disease in the presence and absence of an extrinsic incubation period were compared (Pongsumpun, 2006b). In other study, the effects of there being an incubation period in the virus was studied in a SEIR model (Pongsumpun, 2007). The vector populations in this model were divided into susceptible, infected and infectious classes. In a further study, a seasonal change was introduced into the transmission model used describe the dengue virus infection in Thailand (Pongsumpun, 2011). 
Hiroshi (2006) proposed a new SIR model to clarify the relative contributions of a mathematical approach and of a statistical approach to the dengue epidemiology without having to delve into the mathematical details. He introduced a new method to determine the basic reproductive number which did not involve extensive mathematical manipulations. Erickson et al. (2010) used a SEIR model to examine the role of temperature in driving the vector dynamics. Their model used the Aedes albopictus mosquitoes as the transmitting vector. Bakach and Braselton (2015) looked at different mathematical models and compared their predicted behaviors with each other. The evaluation of each model under different scenarios allowed one to identify the strengths and weakness of each of the model. The effect of the spreading and progression of the disease were studied in order to determine what the values of the parameters were. Rodrigues et al. (2011) has also studied the transmission of dengue fever. They used their results to explain outbreak of the disease in Cape Verde. Aldila et al. (2013) a host-vector dengue transmission model to determine what the optimal control strategy should be. The strategy was based on using different amounts of mosquito repellent on different segments of the human population.

In another paper, Esteva and Vargas (2000) included the possibility of vertical or transovarial transmission. In this type of transmission, one does not need the host; one can pass the virus from mosquito to mosquito without the need of a human or from human to human without the need of a mosquito. These types of transmission are very. The rarity is seen in the report in 2010 of the first putative case of vertical transmission in China (Yin et al., 2010) Thenmozhi et al. (2007) reported that they had collected mosquitoes (both male and female) in Kerala State and examined for dengue virus DNA in them. They found that some of the male mosquitoes in them. Since the males do not need human blood for the purpose of generating eggs, the most likely way would be through sexual contact. This has happen in the most recent ZIKA epidemic (Lequime et al., 2016). The ZIKA disease is also an airborne disease where the same mosquito transmitting the virus is the same virus specie transmitting the dengue fever virus. The latest report is that the ZIKA disease has become a STD (sexually transmitted disease) which needs a different form of disease control strategy. Whatever the reasons, two recent reviews have appeared in 2016 on the vertical transmission of spread of dengue fever (Grunnil and Boots, 2016; D’Ortenzio et al., 2016).

In this study, we will be reconsidering the transmission of dengue virus in the case where vertical transmission of the virus between the mosquitoes is possible. We will be using the SEIR model (Susceptible, Exposed, Infected and Recovered) is used to investigate the dynamics of the disease. We will be analyzing the stability of the model using standard dynamic analysis. We will consider the dynamical transmission model of dengue disease for the cases where vertical transmission is or is not possible. The equilibrium state and stability are considered both behaviors. The numerical simulation, results and conclusion are presented.

\section{Materials and Methods}

\section{Mathematical Model}

The mathematical model is for two populations, human and mosquito. In the SEIR model, the human is divided into four compartments, susceptible human $(\tilde{S} H)$, exposed human $(\tilde{E} H)$, infected human $(\tilde{I} H)$ and re-denoted as covered human $(\tilde{R} H)$. The vector population is partitioned into 3 compartments: Susceptible vector susceptible vector $(\tilde{S} V)$, exposed vector $(\tilde{E} V)$ and infected vector $\left(\tilde{I}_{V}\right)$. In this model, we assumed that the total number of members of each population is constant. For the vector population, we further assume that the rate at which the number of susceptible vectors entering into mosquito population is $A$ per unit time and $M$ is the number of infected mosquitoes that enter directly by vertical (transovarial) infection if this type of transmission is possible. As we have mentioned, this transmission mode is rare or nonexistent. We consider the different behaviors of dynamical transmission of dengue disease with and without the effect of vertical transmission taken into account $(M \neq 0$ and $M=0)$. The dynamic transmission of dengue disease when both modes of transmissions are possible is shown schematically in Fig. 3 .

Let:

$(\tilde{S} H)=$ Number of susceptible humans population at time $t$

$(\tilde{E} H)=$ Number of exposed humans population at time $t$

$(\tilde{I} H)=$ Number of infected humans population at time $t$

$(\tilde{R} H)=$ Number of recovered humans population at time $t$

$(\tilde{S} V)=$ Number of susceptible vector population at time $t$

$(\tilde{E} V)=$ Number of exposed vector population at time $t$

$\left(\tilde{I}_{V}\right)=$ Number of vertically infected population at time $t$

$\mathrm{A}=$ Constant recruitment rate

$\mathrm{M}=$ Number of mosquitoes which were infected transovarially 


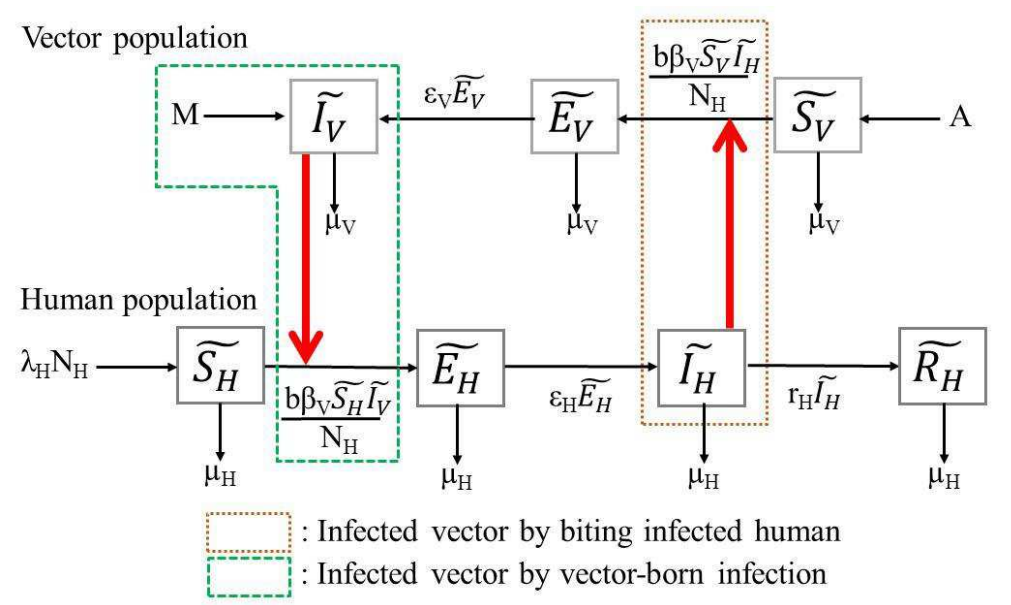

Fig. 3: The dynamic transmission of dengue disease by both biting infected human and vector born infection

The mathematical representations of the processes shown schematically in Fig. 3 are given by equations (1) to (7). The dynamic change of the human and mosquito populations are given by Equation (1) to (4) and (5) to (7) respectively:

$$
\begin{aligned}
& \frac{d \tilde{S}_{H}}{d t}=\lambda_{H} N_{H}-\frac{b \beta_{H}}{N_{H}} \tilde{S}_{H} \tilde{I}_{V}-\mu_{H} \tilde{S}_{H} \\
& \frac{d \tilde{E}_{H}}{d t}=\frac{b \beta_{H}}{N_{H}} \tilde{S}_{H} \tilde{I}_{V}-\left(\varepsilon_{\mathrm{H}}+\mu_{H}\right) \tilde{E}_{H} \\
& \frac{d \tilde{I}_{H}}{d t}=\varepsilon_{H} \tilde{E}_{H}-\left(\mu_{H}+r_{H}\right) \tilde{I}_{H} \\
& \frac{d \tilde{I}_{H}}{d t}=r_{H} \tilde{I}_{H}-\mu_{H} \tilde{R}_{H} \\
& \frac{d \tilde{S}_{V}}{d t}=A-\frac{b \beta_{V}}{N_{H}} \tilde{S}_{V} \tilde{I}_{H}-\mu_{V} \tilde{S}_{V} \\
& \frac{d \tilde{E}_{V}}{d t}=\frac{b \beta_{V}}{N_{H}} \tilde{S}_{V} \tilde{I}_{H}-\left(\varepsilon_{V}+\mu_{V}\right) \tilde{E}_{V} \\
& \frac{d \tilde{I}_{V}}{d t}=M+\varepsilon_{V} \tilde{E}_{V}-\mu_{V} \tilde{I}_{V}
\end{aligned}
$$

We have assumed that:

$$
\begin{aligned}
& N_{H}=\tilde{S}_{H}+\tilde{E}_{H}+\tilde{I}_{H}+\tilde{R}_{H} \\
& N_{V}=\tilde{S}_{V}+\tilde{E}_{V}+\tilde{I}_{V}
\end{aligned}
$$

Where:

$N_{H}=$ Total number of human population

$N_{V}=$ Total number of vector population

$$
\begin{aligned}
\lambda_{H}= & \text { Birth rate of human population } \\
b= & \text { Biting rate of vector population } \\
\beta_{H}= & \text { Transmission probability of dengue virus from } \\
& \text { vector population to human } \\
\beta_{V}= & \text { Transmission probability of dengue virus from } \\
& \text { human population to vector } \\
\varepsilon_{H}= & \text { Intrinsic incubation rate } \\
\varepsilon_{V}= & \text { Extrinsic incubation rate } \\
\mu_{H}= & \text { Death rate of human population } \\
\mu_{V}= & \text { Death rate of vector population } \\
r_{H}= & \text { Recovery rate of the human population }
\end{aligned}
$$

The assumption of our model is the total human and vector populations are constant. This leads to the rate of change for human and vector population being zero, i.e.:

$$
\begin{aligned}
& \frac{d \tilde{S}_{H}}{d t}+\frac{d \tilde{E}_{H}}{d t}+\frac{d \tilde{I}_{H}}{d t}+\frac{d \tilde{R}_{H}}{d t}=0 \\
& \frac{d \tilde{S}_{V}}{d t}+\frac{d \tilde{E}_{V}}{d t}+\frac{d \tilde{I}_{V}}{d t}=0
\end{aligned}
$$

From the above equations, we can obtain the following equations:

$$
\begin{aligned}
& N_{V}=(A+M) / \mu_{V} \\
& \lambda_{H}=\mu_{H}
\end{aligned}
$$

We now normalized equations (1) - (9) as following:

$S_{H}=\frac{\tilde{S}_{H}}{N_{H}}, E_{H}=\frac{\tilde{E}_{H}}{N_{H}}, I_{H}=\frac{\tilde{I}_{H}}{N_{H}}, R_{H}=\frac{\tilde{R}_{H}}{N_{H}}$,

$S_{V}=\frac{\tilde{S}_{V}}{N_{V}}, E_{H}=\frac{\tilde{E}_{V}}{N_{V}}, I_{V}=\frac{\tilde{I}_{V}}{N_{V}}$ 
We also have:

$$
\begin{aligned}
& 1=S_{H}+E_{H}+I_{H}+R_{H} \\
& 1=S_{V}+E_{H}+I_{V}
\end{aligned}
$$

The mathematical model of Equation $(1)-(7)$ is now reduced to the following 5 Equations:

$$
\begin{aligned}
& \frac{d S_{H}}{d t}=\mu_{H}\left(1-S_{H}\right)-\frac{b \beta_{H}}{N_{H}} S_{H} I_{V} N_{V} \\
& \frac{d E_{H}}{d t}=\frac{b \beta_{H}}{N_{H}} S_{H} I_{V} N_{V}-\left(\varepsilon_{H}+\mu_{H}\right) E_{H} \\
& \frac{d I_{H}}{d t}=\varepsilon_{H} E_{H}-\left(\mu_{H}+r_{H}\right) I_{H} \\
& \frac{d E_{V}}{d t}=b \beta_{V} S_{V} I_{H}-\left(\varepsilon_{V}+\mu_{V}\right) E_{V} \\
& \frac{d I_{V}}{d t}=\frac{M}{N_{V}}+\varepsilon_{V} E_{V}-\mu_{V} I_{V}
\end{aligned}
$$

\section{Mathematical Analysis for Equilibrium Point}

The mathematical model is now analyzed and investigated in order to find the equilibrium points and system stability. The equilibrium point is determined by setting the right hand side of Equation (18) - (22) to zero. The system stability is determined by its eigenvalues and $R_{0}$. After we solved Equation (18) (22), we only obtain the endemic disease equilibrium points $E_{l}$ given by:

$$
E_{1}=\left(S_{H}^{1^{*}}, E_{H}^{1^{*}}, I_{H}^{1^{*}}, E_{V}^{1^{*}}, I_{V}^{1^{*}}\right)
$$

Where:

$$
\begin{aligned}
& S_{H}^{1^{*}}=\left(b^{2} N_{V}^{2} \beta_{H} \beta_{V} \varepsilon_{H} \varepsilon_{V} \mu_{H}+\right. \\
& N_{V}\left(N _ { H } \mu _ { H } ( \varepsilon _ { V } + \mu _ { V } ) \left(2 b \beta_{V} \varepsilon_{\mathrm{H}} \mu_{H}+\right.\right. \\
& \left.\left(\gamma_{H}+\mu_{H}\right)\left(\varepsilon_{\mathrm{H}}+\mu_{H}\right) \mu_{V}\right) \\
& +b M \beta_{H}\left(\gamma _ { H } \left(\varepsilon_{\mathrm{H}}+\mu_{H}\left(\varepsilon_{V}+\mu_{V}\right)\right.\right. \\
& \left.\left.+\mu_{H}\left(b \beta_{V} \varepsilon_{\mathrm{H}}+\left(\varepsilon_{\mathrm{H}}+\mu_{H}\right)\left(\varepsilon_{V}+\mu_{V}\right)\right)\right)\right) \\
& -\sqrt{ }\left(N _ { V } ^ { 2 } \left(4 b M N_{H} \beta_{H} \mu_{H}\left(\gamma_{H}+\mu_{H}\right)\left(\varepsilon_{\mathrm{H}}+\mu_{H}\right)\left(\varepsilon_{V}+\mu_{V}\right)^{2}\right.\right. \\
& \left(b \beta_{V} \varepsilon_{\mathrm{H}} \mu_{H}+\left(\gamma_{H}+\mu_{H}\right)\left(\varepsilon_{\mathrm{H}}+\mu_{H}\right) \mu_{V}\right)+ \\
& N_{H} \mu_{H}\left(\gamma_{H}+\mu_{H}\right)\left(\varepsilon_{\mathrm{H}}+\mu_{H}\right) \mu_{V}\left(\varepsilon_{V}+\mu_{V}\right) \\
& -b \beta_{H}\left(M \gamma_{H}\left(\varepsilon_{\mathrm{H}}+\mu_{H}\right)\left(\varepsilon_{V}+\mu_{V}\right)+\right. \\
& \left.\left.\left.\left.\left.\mu_{H}\left(b \beta_{V} \varepsilon_{\mathrm{H}}\left(M+N_{V} \varepsilon_{V}\right)+M\left(\varepsilon_{H}+\mu_{H}\right)\left(\varepsilon_{V}+\mu_{V}\right)\right)\right)\right)^{2}\right)\right)\right) / \\
& \left(2 b N_{V} \beta_{V} \varepsilon_{H} \mu_{H}\left(b \beta_{H}\left(M+N_{V} \varepsilon_{V}\right)+N_{H} \mu_{H}\left(\varepsilon_{V}+\mu_{V}\right)\right)\right)
\end{aligned}
$$

$$
\begin{aligned}
& E_{H}^{1^{*}}=\left(b^{2} N_{V}^{2} \beta_{H} \beta_{V} \varepsilon_{H} \varepsilon_{V} \mu_{H}\right. \\
& +N_{V}\left(-N_{H} \mu_{H}\left(\gamma_{H}+\mu_{H}\right)\left(\varepsilon_{\mathrm{H}}+\mu_{H}\right) \mu_{V}\left(\varepsilon_{V}+\mu_{V}\right)\right. \\
& +b M \beta_{H}\left(-\gamma_{H}\left(\varepsilon_{\mathrm{H}}+\mu_{H}\right)\left(\varepsilon_{V}+\mu_{V}\right)\right. \\
& \left.\left.-\mu_{H}\left(-b \beta_{V} \varepsilon_{\mathrm{H}}+\left(\varepsilon_{\mathrm{H}}+\mu_{H}\right)\left(\varepsilon_{V}+\mu_{V}\right)\right)\right)\right) \\
& +\sqrt{ }\left(N _ { V } ^ { 2 } \left(4 b M N_{H} \beta_{H} \mu_{H}\left(\gamma_{H}+\mu_{H}\right)\right.\right. \\
& \left(\varepsilon_{\mathrm{H}}+\mu_{H}\right)\left(\varepsilon_{V}+\mu_{V}\right)^{2}\left(b \beta_{V} \varepsilon_{\mathrm{H}} \mu_{H}\right. \\
& \left.+\left(\gamma_{H}+\mu_{H}\right)\left(\varepsilon_{\mathrm{H}}+\mu_{H}\right) \mu_{V}\right)+ \\
& N_{H} \mu_{H}\left(\gamma_{H}+\mu_{H}\right)\left(\varepsilon_{\mathrm{H}}+\mu_{H}\right) \mu_{V}\left(\varepsilon_{V}+\mu_{V}\right) \\
& -b \beta_{H}\left(M \gamma_{H}\left(\varepsilon_{\mathrm{H}}+\mu_{H}\right)\left(\varepsilon_{V}+\mu_{V}\right)\right. \\
& \left.\left.\left.\left.\left.+\mu_{H}\left(b \beta_{V} \varepsilon_{\mathrm{H}}\left(M+N_{V} \varepsilon_{V}\right)+M\left(\varepsilon_{H}+\mu_{H}\right)\left(\varepsilon_{V}+\mu_{V}\right)\right)\right)\right)^{2}\right)\right)\right) / \\
& \left(2 b N_{V} \beta_{V} \varepsilon_{H}\left(\varepsilon_{\mathrm{H}}+\mu_{H}\right)\left(b \beta_{H}\left(M+N_{V} \varepsilon_{V}\right)+N_{H} \mu_{H}\left(\varepsilon_{V}+\mu_{V}\right)\right)\right) \\
& I_{H}^{1^{*}}=\left(b^{2} N_{V}^{2} \beta_{H} \beta_{V} \varepsilon_{H} \varepsilon_{V} \mu_{H}+\right. \\
& N_{V}\left(-N_{H} \mu_{H}\left(\gamma_{H}+\mu_{H}\right)\left(\varepsilon_{H}+\mu_{H}\right) \mu_{V}\left(\varepsilon_{V}+\mu_{V}\right)+\right. \\
& +b M \beta_{H}\left(-\gamma_{H}\left(\varepsilon_{H}+\mu_{H}\right)\left(\varepsilon_{V}+\mu_{V}\right)\right. \\
& \left.\left.-\mu_{H}\left(-b \beta_{V} \varepsilon_{H}+\left(\varepsilon_{H}+\mu_{H}\right)\left(\varepsilon_{V}+\mu_{V}\right)\right)\right)\right) \\
& +\sqrt{ }\left(N_{V}^{2} 4 b M N_{H} \beta_{H} \mu_{H}\left(\gamma_{H}+\mu_{H}\right)\left(\varepsilon_{H}+\mu_{H}\right)\left(\varepsilon_{V}+\mu_{V}\right)^{2}\right. \\
& \left(b \beta_{V} \varepsilon_{H} \mu_{H}+\left(\gamma_{H}+\mu_{H}\right)\left(\varepsilon_{H}+\mu_{H}\right) \mu_{V}\right) \\
& +\left(N_{H} \mu_{H}\left(\gamma_{H}+\mu_{H}\right)\left(\varepsilon_{H}+\mu_{H}\right) \mu_{V}\left(\varepsilon_{V}+\mu_{V}\right)\right. \\
& -b \beta_{H}\left(M \gamma_{H}\left(\varepsilon_{H}+\mu_{H}\right)\left(\varepsilon_{V}+\mu_{V}\right)\right. \\
& \left.\left.\left.\left.\left.\left.+\mu_{H}\left(b \beta_{V} \varepsilon_{H}\left(M+N_{V} \varepsilon_{V}\right)+M\left(\varepsilon_{H}+\mu_{H}\right)\left(\varepsilon_{V}+\mu_{V}\right)\right)\right)\right)^{2}\right)\right)\right)\right) / \\
& \left(2 b N_{V} \beta_{V}\left(\gamma_{H}+\mu_{H}\right)\left(\varepsilon_{H}+\mu_{H}\right)\right. \\
& \left.\left(b \beta_{H}\left(M+N_{V} \varepsilon_{V}\right)+N_{H} \mu_{H}\left(\varepsilon_{V}+\mu_{V}\right)\right)\right) \\
& E_{V}^{1^{*}}=\left(-b M N_{V} \beta_{H}\left(\gamma_{H}+\mu_{H}\right)\left(\varepsilon_{H}+\mu_{H}\right) \mu_{V}\left(\varepsilon_{V}+\mu_{V}\right)\right. \\
& -N_{H} N_{V} \mu_{H}\left(\gamma_{H}+\mu_{H}\right)\left(\varepsilon_{H}+\mu_{H}\right) \mu_{V}^{2}\left(\varepsilon_{V}+\mu_{V}\right) \\
& +b^{2} N_{V} \beta_{H} \beta_{V} \varepsilon_{H} \mu_{H}\left(-M \mu_{V}+\varepsilon_{V}\left(-2 M+N_{V} \mu_{V}\right)\right) \\
& +\mu_{V} \sqrt{ }\left(N _ { V } ^ { 2 } \left(4 b M N_{H} \beta_{H} \mu_{H}\left(\gamma_{H}+\mu_{H}\right)\right.\right. \\
& \left(\varepsilon_{H}+\mu_{H}\right)\left(\varepsilon_{V}+\mu_{V}\right)^{2}\left(b \beta_{V} \varepsilon_{H} \mu_{H}\right. \\
& \left.+\left(\gamma_{H}+\mu_{H}\right)\left(\varepsilon_{H}+\mu_{H}\right) \mu_{V}\right)+ \\
& \left(N_{H} \mu_{H}\left(\gamma_{H}+\mu_{H}\right)\left(\varepsilon_{H}+\mu_{H}\right) \mu_{V}\right)\left(\varepsilon_{V}+\mu_{V}\right) \\
& -b \beta_{H}\left(M \gamma_{H}\left(\varepsilon_{H}+\mu_{H}\right)\left(\varepsilon_{V}+\mu_{V}\right)\right. \\
& \left.\left.\left.\left.\left.\left.+\mu_{H} b \beta_{V} \varepsilon_{H}\left(M+N_{V} \varepsilon_{V}\right)+M\left(\varepsilon_{H}+\mu_{H}\right)\left(\varepsilon_{V}+\mu_{V}\right)\right)\right)\right)^{2}\right)\right)\right) / \\
& \left(2 b N_{V}^{2} \beta_{H} \varepsilon_{V}\left(\varepsilon_{V}+\mu_{V}\right)\left(b \beta_{V} \varepsilon_{H} \mu_{H}+\left(\gamma_{H}+\mu_{H}\right)\left(\varepsilon_{H}+\mu_{H}\right) \mu_{V}\right)\right) \\
& I_{V}^{1^{*}}=\left(b^{2} N_{V}^{2} \beta_{H} \beta_{V} \varepsilon_{H} \varepsilon_{V} \mu_{H}+N_{V}\right. \\
& \left(-N_{H} \mu_{H}\left(\gamma_{H}+\mu_{H}\right)\left(\varepsilon_{H}+\mu_{H}\right) \mu_{V}\left(\varepsilon_{V}+\mu_{V}\right)\right. \\
& +b M \beta_{H}\left(\gamma_{H}\left(\varepsilon_{H}+\mu_{H}\right)\left(\varepsilon_{V}+\mu_{V}\right)\right. \\
& \left.\left.+\mu_{H}\left(b \beta_{V} \varepsilon_{H}+\left(\varepsilon_{H}+\mu_{H}\right)\left(\varepsilon_{V}+\mu_{V}\right)\right)\right)\right) \\
& +\sqrt{ }\left(N _ { H } ^ { 2 } \left(4 b M N_{H} \beta_{H} \mu_{H}\left(\gamma_{H}+\mu_{H}\right)\left(\varepsilon_{H}+\mu_{H}\right)\left(\varepsilon_{V}+\mu_{V}\right)^{2}\right.\right. \\
& \left(b \beta_{V} \varepsilon_{H} \mu_{H}+\left(\gamma_{H}+\mu_{H}\right)\left(\varepsilon_{H}+\mu_{H}\right) \mu_{V}\right) \\
& +\left(N_{H} \mu_{H}\left(\gamma_{H}+\mu_{H}\right)\left(\varepsilon_{H}+\mu_{H}\right) \mu_{V}\left(\varepsilon_{V}+\mu_{V}\right)\right. \\
& -b \beta_{H}\left(M \gamma_{H}\left(\varepsilon_{H}+\mu_{H}\right)\left(\varepsilon_{v}+\mu_{v}\right)\right. \\
& \left.\left.\left.\left.\left.+\mu_{H}\left(b \beta_{V} \varepsilon_{H}\left(M+N_{V} \varepsilon_{V}\right)+M\left(\varepsilon_{H}+\mu_{H}\right)\left(\varepsilon_{V}+\mu_{V}\right)\right)\right)\right)^{2}\right)\right)\right) / \\
& \left(2 b N_{V}^{2} \beta_{H}\left(\varepsilon_{V}+\mu_{V}\right)\left(b \beta_{V} \varepsilon_{H} \mu_{H}+\left(\gamma_{H}+\mu_{H}\right)\left(\varepsilon_{H}+\mu_{H}\right) \mu_{V}\right)\right)
\end{aligned}
$$


All parameters used in system (24) - (28) are positive and the epidemic region is:

$$
\Omega=\left\{\left(S_{H}^{1^{*}}, E_{H}^{1^{*}}, I_{H}^{1^{*}}, E_{V}^{1^{*}}, I_{V}^{1^{*}},\right): 0 \leq S_{H}^{1^{*}}, E_{H}^{1^{*}}, I_{H}^{1^{*}}, E_{V}^{1^{*}}, I_{V}^{1^{*}} \leq 1\right\}
$$

\section{Mathematical Analysis for Local Stability}

The local stability of the equilibrium point determined from Equation (18) - (22) is analyzed by first obtaining an expression for the basic reproduction number $R_{0}$ and the Jacobian matrix. After these are done, we then solve the eigenvalues equation which involves the Jacobian matrix. The Jacobian matrix of system (18) $-(22)$ is as follows:

$$
J=\left[\begin{array}{ccccc}
-\mu_{H}-\frac{b \beta_{H}}{N_{H}} I_{V}^{1^{*}} N_{V} & 0 & 0 & 0 & -\frac{b \beta_{H}}{N_{H}} S_{H}^{1^{*}} N_{V} \\
\frac{b \beta_{H}}{N_{H}} I_{V}^{1^{*}} N_{V} & -\left(\varepsilon_{H}+\mu_{H}\right) & 0 & 0 & \frac{b \beta_{H}}{N_{H}} S_{H}^{1^{*}} N_{V} \\
0 & \varepsilon_{H} & -\left(\mu_{H}+r_{H}\right) & 0 & 0 \\
0 & 0 & b \beta_{V}\left(1-I_{V}^{1^{*}}-E_{V}^{1^{*}}\right) & -\left(\varepsilon_{V}+\mu_{V}\right) & 0 \\
0 & 0 & 0 & \varepsilon_{V} & -\mu_{V}
\end{array}\right]
$$

The basic reproductive number, $\sqrt{ } R_{0}$, is the number of secondary infection produced by a typical case of an infection in a population of its infectious period. $R_{0}$ can be indicated the transmission potential of disease. In case of $R_{0}>1$, the transmission has potential to spread between people. The requirement for local stability at the equilibrium state is stated in proposition 1 given below.

\section{Proposition 1.}

The equilibrium state $E_{l}$ is asymptotically stable when $R_{0}$ is higher than $1, R_{0}>1$.

\section{Proof.}

The local stability of $E_{1}$ is governed by linearization of system (18)-(22). The $R_{0}$ is given as:

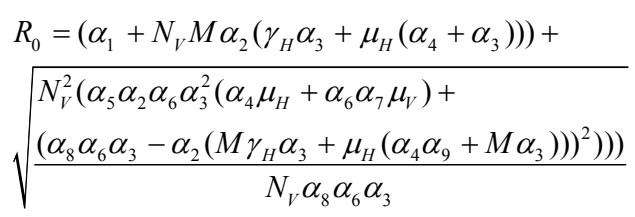

Where:

$$
\begin{aligned}
& a_{1}=b^{2} N_{V}^{2} \beta_{H} \beta_{V} \varepsilon_{H} \varepsilon_{V} \mu_{H} \\
& a_{2}=b \beta_{H} \\
& a_{3}=\left(\varepsilon_{H}+\mu_{H}\right)\left(\varepsilon_{V}+\mu_{V}\right) \\
& a_{4}=b \beta_{V} \varepsilon_{H} \\
& a_{5}=4 N_{H} \mu_{H} M \\
& a_{6}=\left(\gamma_{H}+\mu_{H}\right) \\
& a_{7}=\left(\varepsilon_{H}+\mu_{H}\right) \\
& a_{8}=N_{H} \mu_{H} \mu_{V} \\
& a_{9}=\left(M+N_{V} \varepsilon_{V}\right)
\end{aligned}
$$

The characteristic of equation (29) which determines the eigenvalues is the eigenvalue equation obtained by solving det:

$$
\left|J_{E_{1}}-\lambda I_{5}\right|=0
$$

Where:

$J_{E I}=$ The Jacobian matrix at the equilibrium point $E_{1}$

$\lambda=$ The eigenvalues

$I_{5}=$ The identity $5 \times 5$ matrix

Evaluating the determinant, we get the following Evaluating the determinant, we get the following:

$$
\left(\lambda^{5}+e_{1} \lambda^{4}+e_{2} \lambda^{3}+e_{3} \lambda^{2}+e_{4} \lambda^{1}+e_{5}\right)=0
$$

Where:

$$
\begin{aligned}
& e_{1}=\left[\begin{array}{l}
-F\left(-\gamma_{H}-\mu_{H}\right) \mu_{H}^{2} G H \\
+F\left(-G H J-\mu_{H}^{2} G H\left(-\mu_{H}-K L\right)\right)
\end{array}\right] / N \\
& e_{2}=\left[\begin{array}{l}
\left(-F\left(-\gamma_{H}-\mu_{H}\right) G H J-\mu_{H}^{2} G H\left(-\mu_{H}-K+L\right)\right) \\
\left.+F\left(-G H P+G H J\left(-\mu_{H}-K+L\right)\right)\right)
\end{array}\right] / N \\
& e_{3}=\left[\begin{array}{l}
-F\left(-\gamma_{H}-\mu_{H}\right) G H P+G H J\left(-\mu_{H}-K+L\right) \\
+F O G H+G H P\left(-\mu_{H}-K+L\right)
\end{array}\right] / N \\
& e_{4}=\left[\begin{array}{l}
-F O G H\left(-\mu_{H}-K+L\right)+(Q H(-K+R))(1-K+S) \\
-F\left(\left(-\gamma_{H}-\mu_{H}\right) O G H+G H P\left(-\mu_{H}-K+L\right)\right)
\end{array}\right] / F \mu_{H}^{2} G H \\
& e_{5}=\left(1 / 2 N_{H} N_{V}\left(b \beta_{V} \varepsilon_{H} \mu_{H}+\left(\gamma_{H}+\mu_{H}\right)\left(\varepsilon_{H}+\mu_{H}\right) \mu_{V}\right)\right) T
\end{aligned}
$$

Where:

$$
\begin{aligned}
F= & 8 N_{H}^{2} N_{V}^{4} \beta_{H} \beta_{V}^{2} \varepsilon_{H}^{2} \varepsilon_{V} \\
G= & \left(\varepsilon_{V}+\mu_{V}\right)^{2} \\
H= & \left.\left(b M \beta_{H}+b N_{V} \beta_{H} \varepsilon_{V}\right)+N_{H} \varepsilon_{V} \mu_{H}+N_{H} \mu_{H} \mu_{V}\right)^{2} \\
& \left(b \beta_{V} \varepsilon_{H} \mu_{H}+\gamma_{H} \varepsilon_{H} \mu_{V}+\gamma_{H} \mu_{H} \mu_{V}+\varepsilon_{H} \mu_{H} \mu_{V}+\mu_{H}^{2} \mu_{V}\right)^{3} \\
J= & \left(\left(-\varepsilon_{H}-\mu_{H}\right) \mu_{H}^{2}-\mu_{H}^{2}\left(\varepsilon_{V}+2 \mu_{V}\right)\right) \\
& \left(b^{2} N_{V}^{2} \beta_{H} \beta_{V} \varepsilon_{H} \varepsilon_{V} \mu_{H}\right. \\
& +N_{V}\left(-N_{H} \mu_{H}\left(\gamma_{H}+\mu_{H}\right)\left(\varepsilon_{H}+\mu_{H}\right) \mu_{V}\left(\varepsilon_{V}+\mu_{V}\right)\right. \\
& +b M \beta_{H}\left(\gamma_{H}\left(\varepsilon_{H}+\mu_{H}\right)\left(\varepsilon_{V}+\mu_{V}\right)\right. \\
& \left.\left.+\mu_{H}\left(b \beta_{V} \varepsilon_{H}+\left(\varepsilon_{H}+\mu_{H}\right)\left(\varepsilon_{V}+\mu_{H}\right)\right)\right)\right) \\
& \sqrt{ }\left(N _ { V } ^ { 2 } \left(4 b M N_{H} \beta_{H} \mu_{H}\left(\gamma_{H}+\mu_{H}\right)\left(\varepsilon_{H}+\mu_{H}\right)\left(\varepsilon_{V}+\mu_{V}\right)^{2}\right.\right. \\
& \left(b \beta_{V} \varepsilon_{H} \mu_{H}+\left(\gamma_{H}+\mu_{H}\right)\left(\varepsilon_{H}+\mu_{H}\right) \mu_{V}\right)+ \\
& \left(N_{H} \mu_{H}\left(\gamma_{H}+\mu_{H}\right)\left(\varepsilon_{H}+\mu_{H}\right) \mu_{V}\left(\varepsilon_{V}+\mu_{V}\right)-\right. \\
\mathrm{L}= & b \beta_{H}\left(M \gamma_{H}\left(\varepsilon_{H}+\mu_{H}\right)\left(\varepsilon_{V}+\mu_{V}\right)\right. \\
& \left.\left.\left.\left.\left.+\mu_{H}\left(b \beta_{V} \varepsilon_{H}\left(M+N_{V} \varepsilon_{V}\right)+M\left(\varepsilon_{H}+\mu_{H}\right)\left(\varepsilon_{V}+\mu_{V}\right)\right)\right)\right)^{2}\right)\right)\right) / \\
& \left.\left.\left(2 N_{H} N_{V}\left(\varepsilon_{V}+\mu_{V}\right)\left(b \beta_{V} \varepsilon_{H} \mu_{H}+\left(\gamma_{H}+\mu_{H}\right)\left(\varepsilon_{H}+\mu_{H}\right)\right)\right)\right)\right) \\
& \left(8 N_{H}^{2} N_{V}^{4} \beta_{V} \beta_{V}^{2} \varepsilon_{H}^{2} \varepsilon_{H}^{2}\left(\varepsilon_{V}+\mu_{V}\right)^{2}\right. \\
\mathrm{N}= & \left(b M \beta_{H}+b N_{V} \beta_{H} \varepsilon_{V}+N_{H} \varepsilon_{V} \mu_{H}+N_{H} \mu_{H} \mu_{V}\right)^{2} \\
\mathrm{O}= & \left.\left(b \beta_{V} \varepsilon_{H} \mu_{H}+\gamma_{H} \varepsilon_{H} \mu_{V}+\gamma_{H} \mu_{H} \mu_{V}+\varepsilon_{H} \mu_{H} \mu_{V}+\mu_{H}^{2} \mu_{V}\right)^{3}\right) \\
& \left(-\varepsilon_{H}\right) \mu_{H}^{2}\left(-\varepsilon_{V}-\mu_{V}\right) \mu_{V} \\
= & \\
= &
\end{aligned}
$$




$$
\begin{aligned}
& \mathrm{P}=\left(\mu_{H}^{2}\left(-\varepsilon_{V}-\mu_{V}\right) \mu_{V}+\left(-\varepsilon_{H}-\mu_{H}\right) \mu_{H}^{2}\left(\varepsilon_{V}+2 \mu_{V}\right)\right) \\
& \mathrm{Q}=1 /\left(b \beta_{H}\left(M+N_{V} \varepsilon_{V}\right)+N_{H} \mu_{H}\left(\varepsilon_{V}+\mu_{V}\right)\right) \\
& 4 b N_{H} N_{V}^{4} \beta_{H}^{2} \beta_{V}^{2} \varepsilon_{H}^{2} \varepsilon_{V}^{2} \mu_{H}\left(\varepsilon_{V}+\mu_{V}\right)^{2} \\
& \sqrt{ }\left(N _ { V } ^ { 2 } \left(4 b M N_{H} \beta_{H} \mu_{H}\left(\gamma_{H}+\mu_{H}\right)\left(\varepsilon_{H}+\mu_{H}\right)\left(\varepsilon_{V}+\mu_{V}\right)^{2}\right.\right. \\
& \left.b \beta_{V} \varepsilon_{H} \mu_{H}+\left(\gamma_{H}+\mu_{H}\right)\left(\varepsilon_{H}+\mu_{H}\right) \mu_{V}\right) \\
& \mathrm{S}=+\left(N_{H} \mu_{H}\left(\gamma_{H}+\mu_{H}\right)\left(\varepsilon_{H}+\mu_{H}\right) \mu_{V}\left(\varepsilon_{V}+\mu_{V}\right)\right. \\
& -b \beta_{H}\left(M \gamma_{H}\left(\varepsilon_{H}+\mu_{H}\right)\left(\varepsilon_{V}+\mu_{V}\right)\right. \\
& \left.\left.\left.\left.\left.+\mu_{H}\left(b \beta_{V} \varepsilon_{H}\left(M+N_{V} \varepsilon_{V}\right)+M\left(\varepsilon_{H}+\mu_{H}\right)\left(\varepsilon_{V}+\mu_{V}\right)\right)\right)\right)^{2}\right)\right)\right) \\
& /\left(2 b N_{V}^{2} \beta_{H}\left(\varepsilon_{V}+\mu_{V}\right)\left(b \beta_{V} \varepsilon_{H} \mu_{H}+\left(\gamma_{H}+\mu_{H}\right)\left(\varepsilon_{H}+\mu_{H}\right) \mu_{V}\right)\right) \\
& =\left(-b^{3} N_{V}^{2} \beta_{H} \beta_{V}^{2} \varepsilon_{H}^{2} \varepsilon_{V} \mu_{H}^{2}\right. \\
& +b N_{V} \beta_{V} \varepsilon_{H} \mu_{H}\left(N_{H} \mu_{H}\left(\gamma_{H}+\mu_{H}\right)\left(\varepsilon_{H}+\mu_{H}\right) \mu_{V}\left(\varepsilon_{V}+\mu_{V}\right)\right. \\
& +b M \beta_{H}\left(-\gamma_{H}\left(\varepsilon_{H}+\mu_{H}\right)\left(\varepsilon_{V}+\mu_{V}\right)\right. \\
& \left.\left.-\mu_{H}\left(b \beta_{V} \varepsilon_{H}+\left(\varepsilon_{H}+\mu_{H}\right)\left(\varepsilon_{V}+\mu_{V}\right)\right)\right)\right) \\
& +\left(b \beta_{V} \varepsilon_{H} \mu_{H}+2\left(\gamma_{H}+\mu_{H}\right)\left(\varepsilon_{H}+\mu_{H}\right) \mu_{V}\right) \\
& \mathrm{T}=\sqrt{ }\left(N _ { V } ^ { 2 } \left(N_{H}^{2} \mu_{H}^{2}\left(\gamma_{H}+\mu_{H}\right)^{2}\left(\varepsilon_{H}+\mu_{H}\right)^{2} \mu_{V}^{2}\left(\varepsilon_{V}+\mu_{V}\right)^{2}\right.\right. \\
& +2 b N_{H} \beta_{H} \mu_{H}\left(\gamma_{H}+\mu_{H}\left(\varepsilon_{H}+\mu_{H}\right)\left(\varepsilon_{V}+\mu_{V}\right)\right. \\
& \left(M\left(\gamma_{H}+\mu_{H}\right)\left(\varepsilon_{H}+\mu_{H}\right) \mu_{V}\left(\varepsilon_{V}+\mu_{V}\right)\right. \\
& +b \beta_{V} \varepsilon_{H} \mu_{H}\left(2 M \varepsilon_{V}+\left(M-N_{V} \varepsilon_{V}\right) \mu_{V}\right) \\
& +b^{2} \beta_{H}^{2}\left(M \gamma_{H}\left(\varepsilon_{H}+\mu_{H}\right)\left(\varepsilon_{V}+\mu_{V}\right)\right. \\
& \left.\left.\left.\left.+\mu_{H}\left(b \beta_{V} \varepsilon_{H}\left(M+N_{V} \varepsilon_{V}\right)+M\left(\varepsilon_{H}+\mu_{H}\right)\left(\varepsilon_{V}+\mu_{V}\right)\right)\right)^{2}\right)\right)\right) \\
& \sqrt{ }\left(N _ { V } ^ { 2 } \left(4 b M N_{H} \beta_{H} \mu_{H}\left(\gamma_{H}+\mu_{H}\right)\left(\varepsilon_{H}+\mu_{H}\right)\left(\varepsilon_{V}+\mu_{V}\right)^{2}\right.\right. \\
& \left(b \beta_{V} \varepsilon_{H} \mu_{H}+\left(\gamma_{H}+\mu_{H}\right)\left(\varepsilon_{H}+\mu_{H}\right) \mu_{V}\right) \\
& \mathrm{R}=+\left(N_{H} \mu_{H}\left(\gamma_{H}+\mu_{H}\right)\left(\varepsilon_{H}+\mu_{H}\right) \mu_{V}\left(\varepsilon_{V}+\mu_{V}\right)\right. \\
& -b \beta_{H}\left(M \gamma_{H}\left(\varepsilon_{H}+\mu_{H}\right)\left(\varepsilon_{V}+\mu_{V}\right)\right. \\
& \left.\left.\left.\left.\left.+\mu_{H}\left(b \beta_{V} \varepsilon_{H}\left(M+N_{V} \varepsilon_{V}\right)+M\left(\varepsilon_{H}+\mu_{H}\right)\left(\varepsilon_{V}+\mu_{V}\right)\right)\right)\right)^{2}\right)\right)\right)
\end{aligned}
$$

The solution of equation (31) is solved through use of the Routh-Hurwitz criteria. The equilibrium point will be local stability when all eigenvalues have negative real parts. This will happen if all the coefficients satisfy the following conditions:

$$
\begin{aligned}
& e_{1}>0, e_{2}>0, e_{3}>0, e_{4}>0, e_{5}>0 \\
& e_{1} e_{2} e_{3}>e_{3}^{2}+e_{1}^{2} e_{4} \\
& \left(e_{1} e_{4}-e_{5}\right)\left(e_{1} e_{2} e_{3}-e_{3}^{2}-e_{1}^{2} e_{4}\right)>e_{5}\left(e_{1} e_{2}-e_{3}\right)^{2}+e_{1} e_{5}
\end{aligned}
$$

All conditions of equation (32) - (34) are satisfied for endemic equilibrium point as seen in Fig. 4.

The dynamic transmission of dengue disease without a vertical mode of transmission $(M=0)$ is described by equations (18) to (21) which are the same as the case where vertical transmission is possible except that Equation (22) has been replaced by the equation below:

$$
\frac{d I_{V}}{d t}=\varepsilon_{V} E_{V}-\mu_{V} I_{V}
$$

Reanalyzing the new set of equations in the same way as before, we now arrive at two equilibrium points, a disease free equilibrium point and an endemic disease equilibrium point defined as.

i. Disease free equilibrium point

$$
E_{0}=(1,0,0,0,0)
$$

ii. Endemic disease equilibrium point:

$$
E_{2}=\left(S_{H}^{2^{*}}, E_{H}^{2^{*}}, I_{H}^{2^{*}}, E_{V}^{2^{*}}, I_{V}^{2^{*}}\right)
$$

Where:

$S_{H}^{2^{*}}=\left[N_{H}\left(\varepsilon_{V}+\mu_{V}\right)\left(b \beta_{V} \varepsilon_{H} \mu_{H}+\left(\gamma_{H}+\mu_{H}\right)\left(\varepsilon_{H}+\mu_{H}\right) \mu_{V}\right)\right]$
$/\left[b \beta_{V} \varepsilon_{H}\left(b N_{V} \beta_{H} \varepsilon_{V}+N_{H} \mu_{H}\left(\varepsilon_{V}+\mu_{V}\right)\right)\right]$

$E_{H}^{2^{*}}=$

$-\left[\mu_{H}\left(-b^{2} N_{V} \beta_{H} \beta_{V} \varepsilon_{H} \varepsilon_{V}+N_{H}\left(\gamma_{H}+\mu_{H}\right)\left(\varepsilon_{H}+\mu_{H}\right) \mu_{V}\left(\varepsilon_{V}+\mu_{V}\right)\right)\right]$

$/\left[b \beta_{V} \varepsilon_{H}\left(\varepsilon_{H}+\mu_{H}\right)\left(b N_{V} \beta_{H} \varepsilon_{V}+N_{H} \mu_{H}\left(\varepsilon_{V}+\mu_{V}\right)\right)\right]$

$I_{H}^{2^{*}}=\left[\begin{array}{l}\mu_{H}\left(b^{2} N_{V} \beta_{H} \beta_{V} \varepsilon_{H} \varepsilon_{V}\right. \\ \left.-N_{H}\left(\gamma_{H}+\mu_{H}\right)\left(\varepsilon_{H}+\mu_{H}\right) \mu_{V}\left(\varepsilon_{V}+\mu_{V}\right)\right)\end{array}\right]$

$/ b \beta_{V}\left(\gamma_{H}+\mu_{H}\right)\left(\varepsilon_{H}+\mu_{H}\right)\left(b N_{V} \beta_{H} \varepsilon_{V}+N_{H} \mu_{H}\left(\varepsilon_{V}+\mu_{V}\right)\right)$

$E_{V}^{2^{*}}=-\left[\mu_{H} \mu_{V}\left(-b^{2} N_{V} \beta_{H} \beta_{V} \varepsilon_{H} \varepsilon_{V}\right.\right.$

$\left.\left.+N_{H}\left(\gamma_{H}+\mu_{H}\right)\left(\varepsilon_{H}+\mu_{H}\right) \mu_{V}\left(\varepsilon_{V}+\mu_{V}\right)\right)\right]$

$/ b N_{V} \beta_{H} \varepsilon_{V}\left(\varepsilon_{V}+\mu_{V}\right)\left(b \beta_{V} \varepsilon_{H} \mu_{H}+\left(\gamma_{H}+\mu_{H}\right)\left(\varepsilon_{H}+\mu_{H}\right) \mu_{V}\right)$

$I_{V}^{2^{*}}=$

$-\left[\mu_{H}\left(-b^{2} N_{V} \beta_{H} \beta_{V} \varepsilon_{H} \varepsilon_{V}+N_{H}\left(\gamma_{H}+\mu_{H}\right)\left(\varepsilon_{H}+\mu_{H}\right) \mu_{V}\left(\varepsilon_{V}+\mu_{V}\right)\right)\right]$

lbN $\beta_{V} \beta_{H}\left(\varepsilon_{V}+\mu_{V}\right)\left(b \beta_{V} \varepsilon_{H} \mu_{H}+\left(\gamma_{H}+\mu_{H}\right)\left(\varepsilon_{H}+\mu_{H}\right) \mu_{V}\right)$

The Jacobian matrix used to determine the stability of eigenvalues at $\mathrm{E}_{0}=(1,0,0,0,0)$ has the form:

$J=\left[\begin{array}{ccccc}-\mu_{H} & 0 & 0 & 0 & -\frac{b \beta_{H}}{N_{H}} N_{V} \\ 0 & -\left(\varepsilon_{H}+\mu_{H}\right) & 0 & 0 & -\frac{b \beta_{H}}{N_{H}} N_{V} \\ 0 & \varepsilon_{H} & -\left(\mu_{H}+r_{H}\right) & 0 & 0 \\ 0 & 0 & b \beta_{V} & -\left(\varepsilon_{V}+\mu_{V}\right) & 0 \\ 0 & 0 & 0 & \varepsilon_{V} & -\mu_{V}\end{array}\right]$

\section{Proposition 2.}

The equilibrium state $E_{0}$ is asymptotically stable when $R_{0}$ is less than $1, R_{0}<1$. 

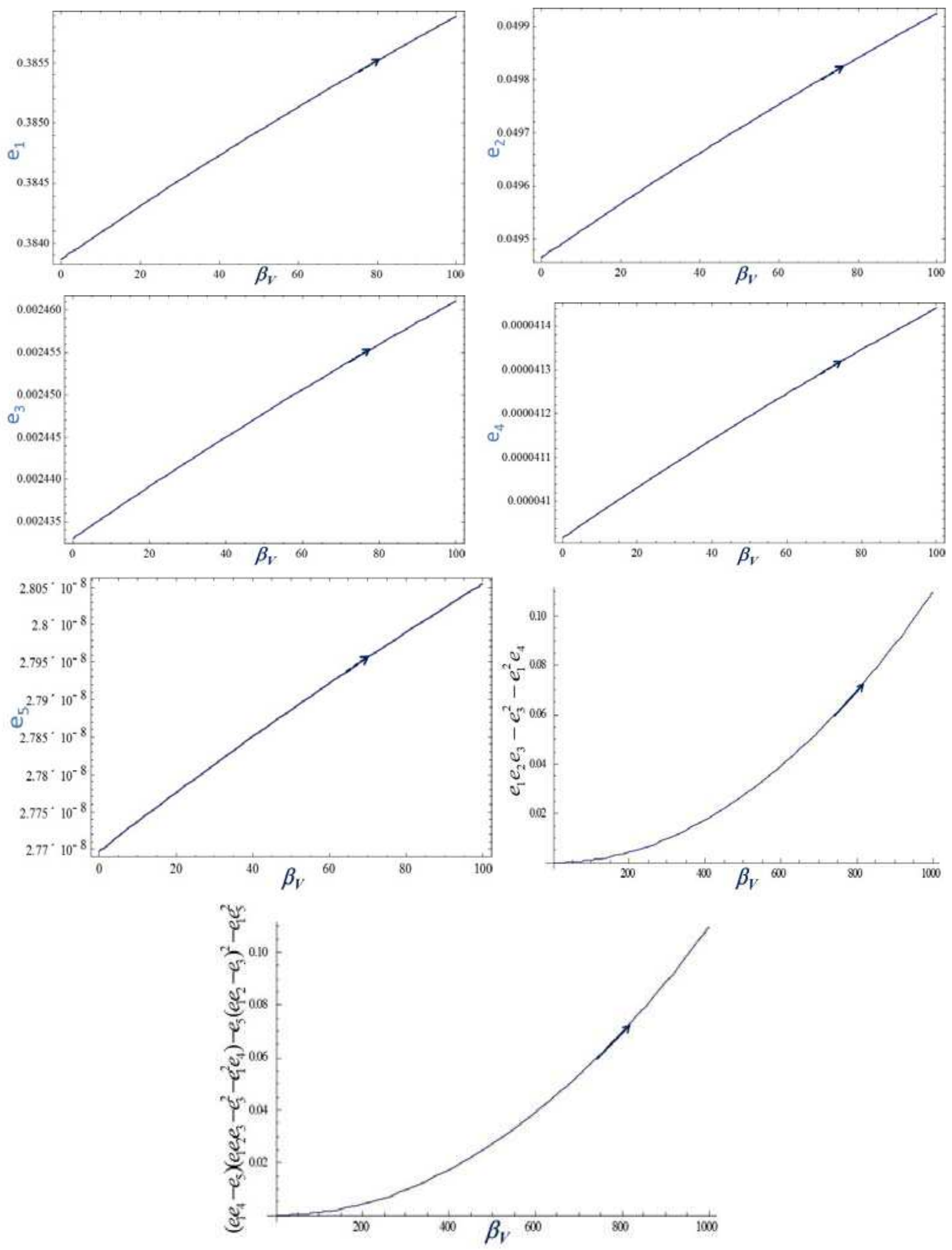

Fig. 4: The All parameters spaces of endemic disease equilibrium of $E_{1}$ are satisfied the Routh-Hurwitz criteria. The parameter value are $N_{H}$ $=92,000, \mathrm{~b}=1 / 5, \mu_{H}=1 /\left(70^{*} 365\right) \gamma_{H}=0.1428, \beta_{H}=0.95, \beta_{V}=0.75, \mu_{V}=1 / 24, \varepsilon_{V}=0.1428, \varepsilon_{H}=0.1667, \mathrm{~A}=5,000$ and $\mathrm{M}=400$ 


\section{Proof.}

The local stability of $E_{0}$ is governed by linearization of system (18) - (21) and (35). The $R_{0}$ will be of the form:

$$
R_{0}=\frac{b^{2} N_{V} \beta_{H} \beta_{V} \varepsilon_{H} \varepsilon_{V}}{N_{H} \mu_{V}\left(\gamma_{H}+\mu_{H}\right)\left(\varepsilon_{H}+\mu_{H}\right)\left(\varepsilon_{V}+\mu_{V}\right)}
$$

The characteristic equation obtained same by solving the determinant equation, Equation (43) is given by:

$$
\left(\lambda^{5}+e_{1} \lambda^{4}+e_{2} \lambda^{3}+e_{3} \lambda^{2}+e_{4} \lambda^{1}+e_{5}\right)=0
$$

Where:

$$
\begin{aligned}
e_{1}= & {\left[N_{H} \gamma_{H}+N_{H} \varepsilon_{H}+N_{H} \varepsilon_{V}+3 N_{H} \mu_{H}+2 N_{H} \mu_{V}\right] / N_{H} } \\
& {\left[N_{H} \gamma_{H} \varepsilon_{H}+N_{H} \gamma_{H} \varepsilon_{V}+N_{H} \varepsilon_{H} \varepsilon_{V}+2 N_{H} \gamma_{H} \mu_{H}+2 N_{H} \varepsilon_{H} \mu_{H}\right.} \\
e_{2}= & +3 N_{H} \varepsilon_{V} \mu_{H}+3 N_{H} \mu_{H}^{2}+2 N_{H} \gamma_{H} \mu_{V}+2 N_{H} \varepsilon_{H} \mu_{V}+N_{H} \varepsilon_{V} \mu_{V} \\
& \left.+6 N_{H} \mu_{H} \mu_{V}+N_{H} \mu_{V}^{2}\right] / N_{H} \\
& {\left[\left(N_{H} \gamma_{H} \varepsilon_{H} \varepsilon_{V}+N_{H} \gamma_{H} \varepsilon_{H} \mu_{H}+2 N_{H} \gamma_{H} \varepsilon_{V} \mu_{H}+2 N_{H} \varepsilon_{H} \varepsilon_{V} \mu_{H}\right.\right.} \\
& +N_{H} \gamma_{H} \mu_{H}^{2}+N_{H} \varepsilon_{H} \mu_{H}^{2}+3 N_{H} \varepsilon_{V} \mu_{V}^{2}+N_{H} \mu_{V}^{3}+2 N_{H} \gamma_{H} \varepsilon_{\varepsilon_{H}} \mu_{V} \\
e_{3}= & +N_{H} \gamma_{H} \varepsilon_{V} \mu_{V}+N_{H} \varepsilon_{H} \varepsilon_{V} \mu_{V}+4 N_{H} \gamma_{H} \mu_{H} \mu_{V}+4 N_{H} \varepsilon_{H} \mu_{H} \mu_{V} \\
& +3 N_{H} \varepsilon_{V} \mu_{H} \mu_{V}+6 N_{H} \mu_{H}^{2} \mu_{V}+N_{H} \gamma_{H} \mu_{V}^{2} \\
& \left.+N_{H} \varepsilon_{H} \mu_{V}^{2}+3 N_{H} \mu_{H} \mu_{V}^{2}\right] / N_{H} \\
& {\left[-b^{2} N_{V} \beta_{H} \beta_{V} \varepsilon_{H} \varepsilon_{V}+N_{H} \gamma_{H} \varepsilon_{H} \varepsilon_{V} \mu_{H}+N_{H} \gamma_{H} \varepsilon_{V} \mu_{H}^{2}\right.} \\
& +N_{H} \varepsilon_{H} \varepsilon_{V} \mu_{H}^{2}+N_{H} \varepsilon_{V} \mu_{H}^{3}+N_{H} \gamma_{H} \varepsilon_{H} \varepsilon_{V} \mu_{V}+2 N_{H} \gamma_{H} \varepsilon_{H} \mu_{H} \mu_{V} \\
e_{4}= & +2 N_{H} \gamma_{H} \varepsilon_{V} \mu_{H} \mu_{V}+2 N_{H} \varepsilon_{H} \varepsilon_{V} \mu_{H} \mu_{V}+2 N_{H} \gamma_{H} \mu_{H}^{2} \mu_{V} \\
& +2 N_{H} \varepsilon_{H} \mu_{H}^{2} \mu_{V}+3 N_{H} \varepsilon_{V} \mu_{H}^{2} \mu_{V}+2 N_{H} \mu_{H}^{3} \mu_{V}+N_{H} \gamma_{H} \varepsilon_{H} \mu_{V}^{2} \\
& \left.\left.+2 N_{H} \gamma_{H} \mu_{H} \mu_{V}^{2}+2 N_{H} \varepsilon_{H} \mu_{H} \mu_{V}^{2}+3 N_{H} \mu_{H}^{2} \mu_{V}^{2}\right)\right] / N_{H}
\end{aligned}
$$
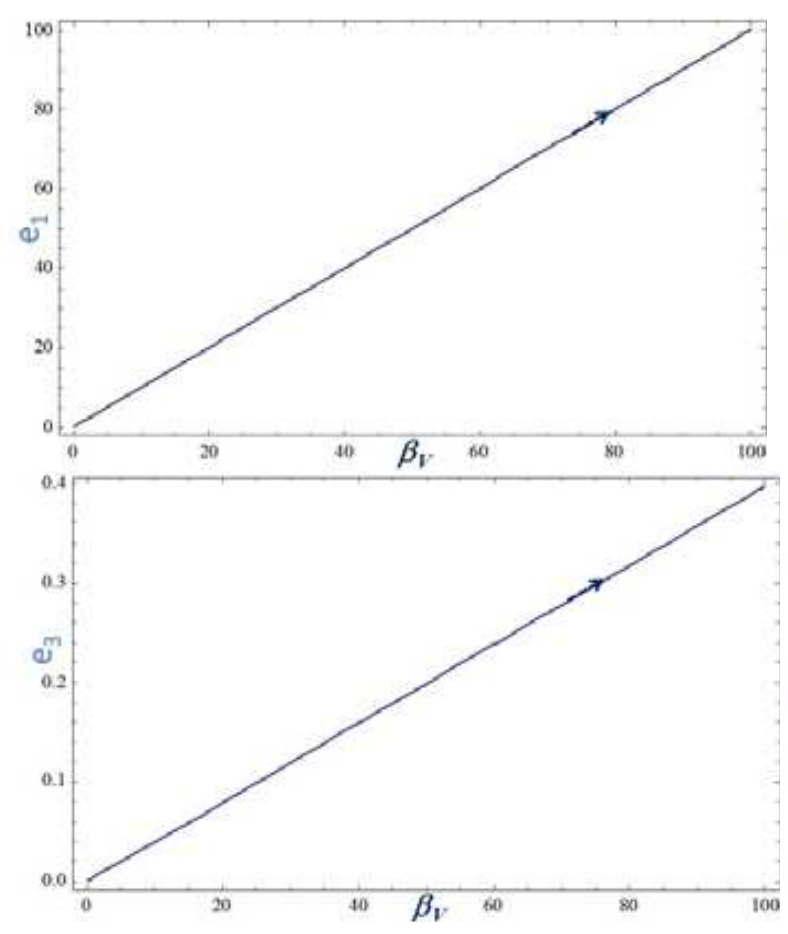

$$
\begin{aligned}
e_{5}= & {\left[\mu _ { H } \left(-b^{2} N_{V} \beta_{H} \beta_{V} \varepsilon_{H} \varepsilon_{V}\right.\right.} \\
& \left.\left.+N_{H}\left(\gamma_{H}+\mu_{H}\right)\left(\varepsilon_{H}+\mu_{H}\right) \mu_{V}\left(\varepsilon_{V}+\mu_{V}\right)\right)\right] / N_{H}
\end{aligned}
$$

All conditions of equation (32)-(34) are satisfied for disease free equilibrium point as seen in Fig. 5.

The Jacobian matrix at $E_{2}=\left(S_{H}^{2^{*}}, E_{H}^{2^{*}}, I_{H}^{2^{*}}, E_{V}^{2^{*}}, I_{V}^{2^{*}}\right)$ is:

$$
J=\left[\begin{array}{ccccc}
-\mu_{H}-\frac{b \beta_{H}}{N_{H}} I_{V}^{2^{*}} N_{V} & 0 & 0 & 0 & -\frac{b \beta_{H}}{N_{H}} S_{H}^{2^{*}} N_{V} \\
\frac{b \beta_{H}}{N_{H}} I_{V}^{2^{+*}} N_{V} & -\left(\varepsilon_{H}+\mu_{H}\right) & 0 & 0 & -\frac{b \beta_{H}}{N_{H}} S_{H}^{2^{*}} N_{V} \\
0 & \varepsilon_{H} & -\left(\mu_{H}+r_{H}\right) & 0 & 0 \\
0 & 0 & b \beta_{V}\left(1-I_{V}^{2 *}-E_{V}^{2 *}\right) & -\left(\varepsilon_{V}+\mu_{V}\right) & 0 \\
0 & 0 & 0 & \varepsilon_{V} & -\mu_{V}
\end{array}\right]
$$

\section{Proposition 3.}

The equilibrium state $E_{2}$ is asymptotically stable when $R_{0}$ is higher than $1, R_{0}>1$.

\section{Proof.}

The local stability of E2 is established through the linearization of equations (18) - (21) and (35) which leads to the determinant equation previously obtained. Solving the eigen value equation, we get a similar characteristic equation, i.e:

$$
\left(\lambda^{5}+e_{1} \lambda^{4}+e_{2} \lambda^{3}+e_{3} \lambda^{2}+e_{4} \lambda^{1}+e_{5}\right)=0
$$

Except that the coefficients are now.

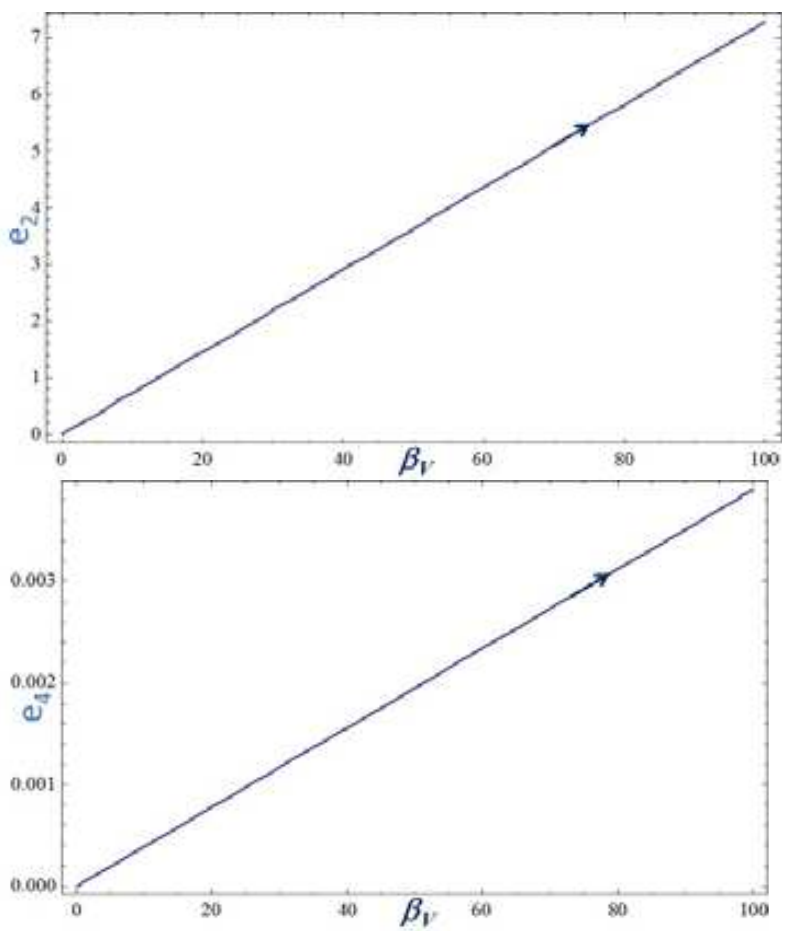




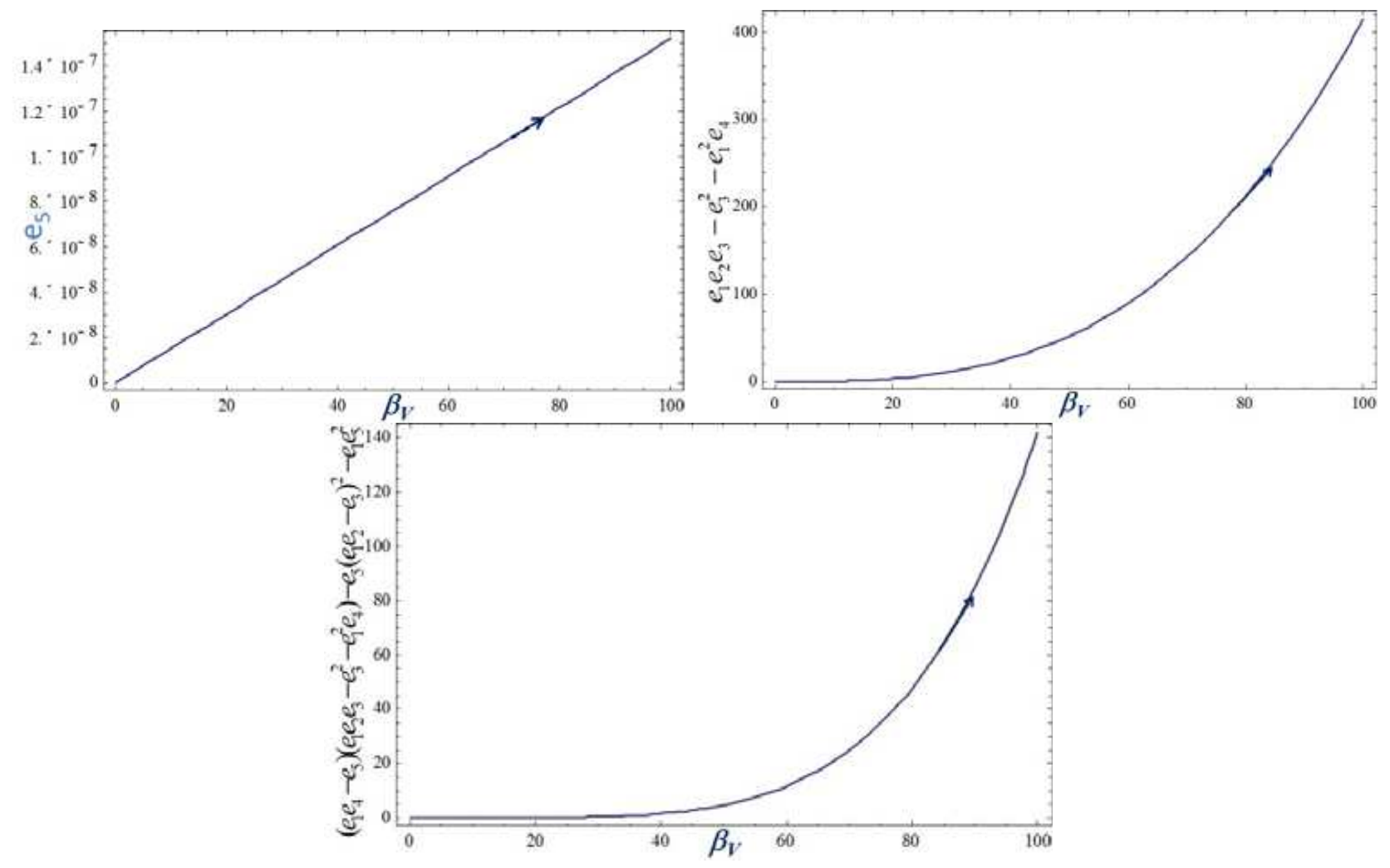

Fig. 5: The All parameters spaces of endemic disease equilibrium of $E 0$ are satisfied the Routh-Hurwitz criteria. The parameter value are $N_{H}=92,000, \mathrm{~b}=1 / 5, \mu_{H}=1 /(70 * 365), \gamma_{H}=0.01428, \beta_{H}=0.65, \beta_{V}=0.65, \mu_{V}=1 / 24, \varepsilon_{V}=0.01428, \varepsilon_{H}=0.1667, A$ $=5,000$ and $M=400$
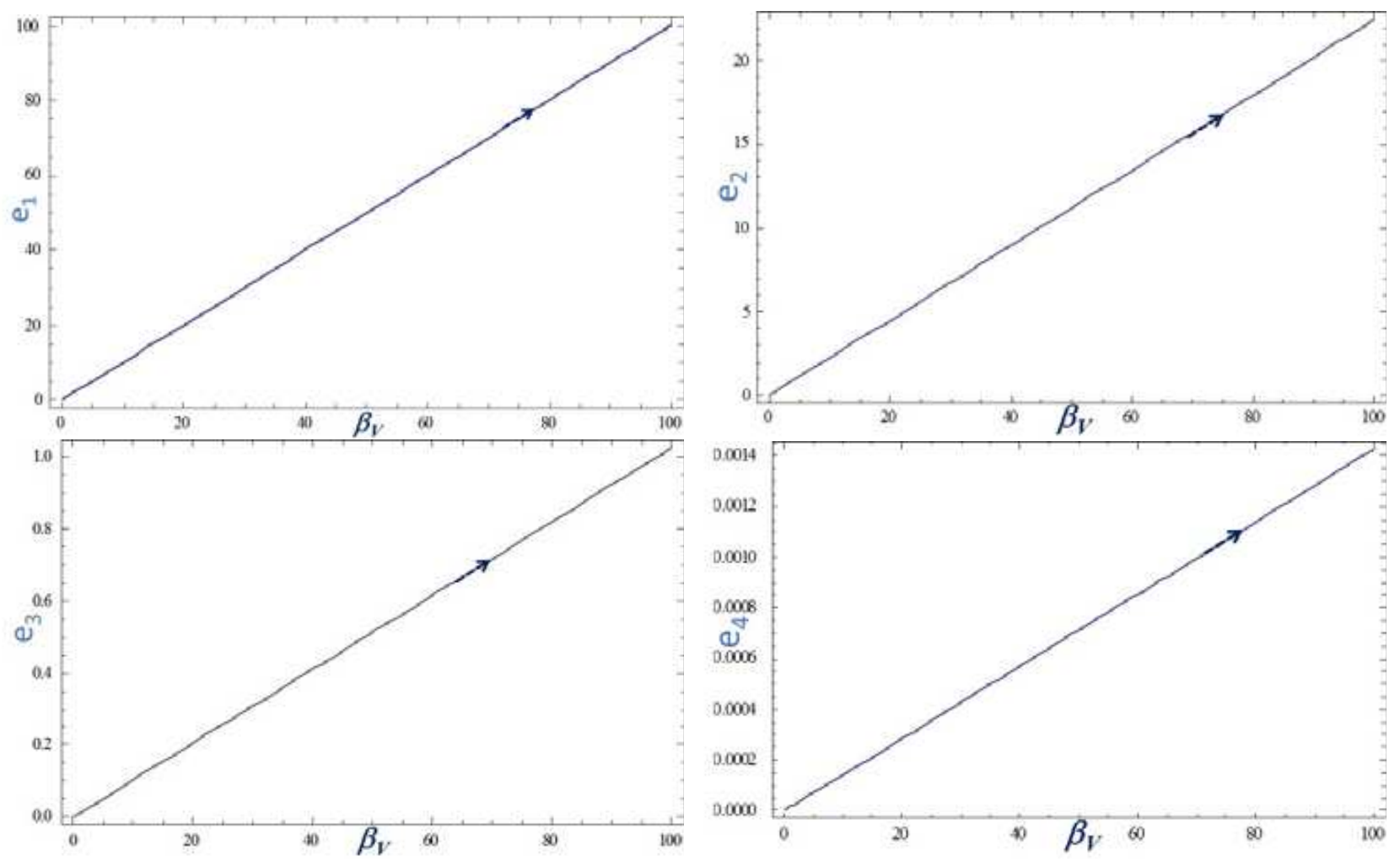


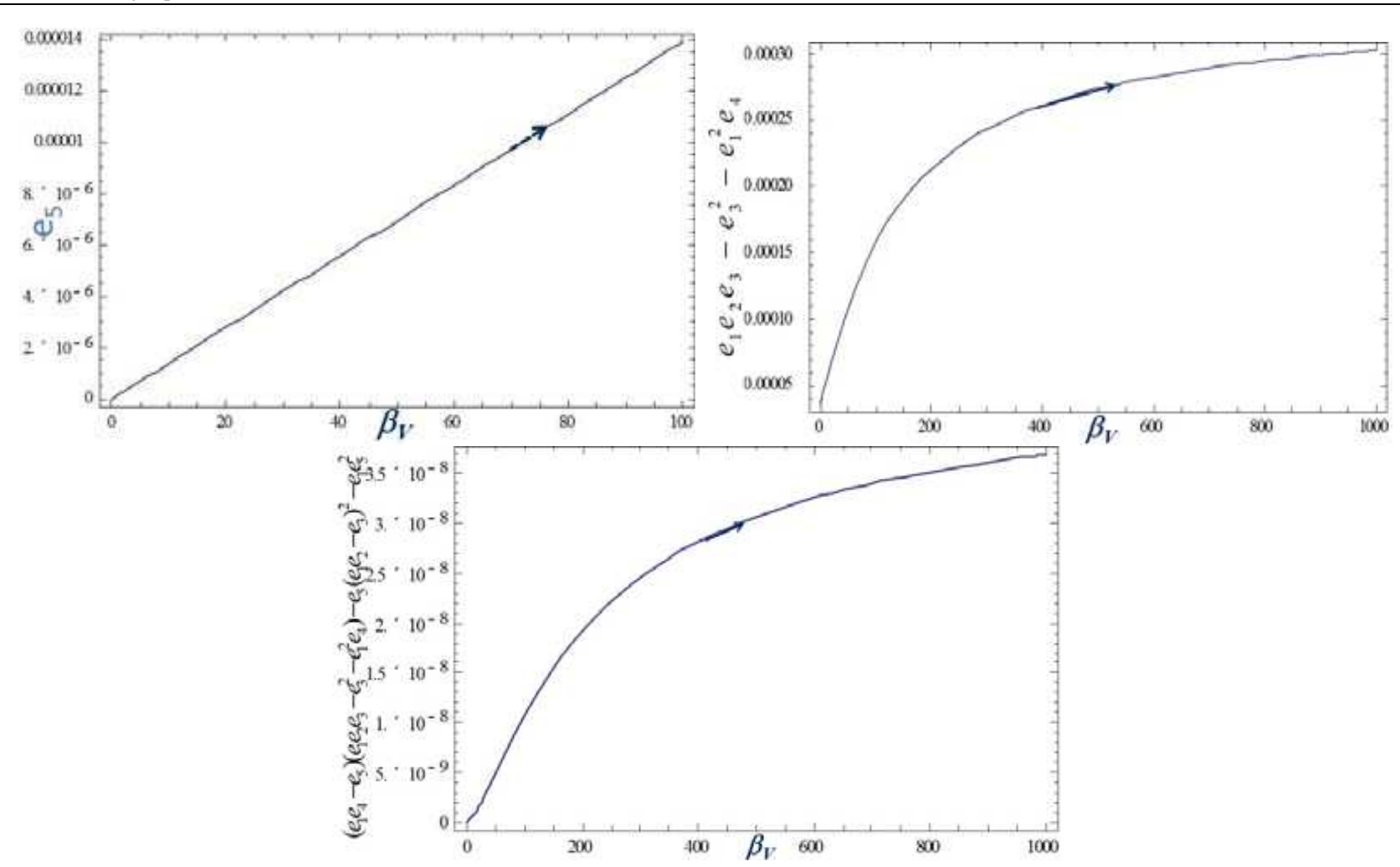

Fig. 6: The All parameters spaces of endemic disease equilibrium of $\mathrm{E} 2$ are satisfied the Routh-Hurwitz criteria. The parameter value are $N_{H}$ $=92,000, b=1 / 5, \mu_{H}=1 /(70 * 365), \gamma_{H}=0.01428, \beta_{H}=0.65, \beta_{V}=0.65, \mu_{V}=1 / 24, \varepsilon_{H}=0.1428, \varepsilon_{H}=0.1667, A=5,000$ and $M=0$

Where:

$$
\begin{aligned}
e_{1}= & {\left[-\left(-U H+G H\left(-\mu_{H}+V\right)\right] / G H\right.} \\
e_{2}= & {\left[-\left(-G H\left(W_{1}-W_{2}+W_{3} W_{4}\right)+G U H\left(-\mu_{H}+V\right)\right] / G H\right.} \\
e_{3}= & {\left[-\left(-U H\left(-W_{3} W_{2}+W_{1} W_{4}\right)\right.\right.} \\
& \left.+G H\left(W_{1}-W_{2}+W_{3} W_{4}\right)\left(-\mu_{H}+V\right)\right] / G H \\
e_{4}= & {\left[\left(1 / Y_{1} H Y_{2}\right)\left(1+V+\mu_{V} V\right)-Y_{3}\left(W_{1} W_{2} G H\right.\right.} \\
& \left.\left.+G H\left(-W_{3} W_{2}+W_{1} W_{4}\right)\right)\left(-\mu_{H}+V\right)\right] / G H Y_{3} \\
& {\left[-\mu_{H}\left(\gamma_{H}+\mu_{H}\right)\left(\varepsilon_{H}+\mu_{H}\right) \mu_{V}\left(-b^{2} N_{V} \beta_{H} \beta_{V} \varepsilon_{H} \varepsilon_{V}\right.\right.} \\
& \left.\left.+N_{H}\left(\gamma_{H}+\mu_{H}\right)\left(\varepsilon_{H}+\mu_{H}\right) \mu_{V}\left(\varepsilon_{V}+\mu_{V}\right)\right)\right] \\
& / N_{H}\left(b \beta_{V} \varepsilon_{H} \mu_{H}+\left(\gamma_{H}+\mu_{H}\right)\left(\varepsilon_{H}+\mu_{H}\right) \mu_{V}\right)
\end{aligned}
$$

Where:

$$
\begin{aligned}
\mathrm{U}= & \left(\gamma_{H}+\varepsilon_{H}+\varepsilon_{V}+2 \mu_{H}+2 \mu_{V}\right) \\
V= & {\left[\mu_{H}\left(-b^{2} N_{V} \beta_{H} \beta_{V} \varepsilon_{H} \varepsilon_{V}+N_{H}\left(\gamma_{H}+\mu_{H}\right)\left(\varepsilon_{H}+\mu_{H}\right) \mu_{V}\left(\varepsilon_{V}+\mu_{V}\right)\right)\right] } \\
& /\left[N_{H}\left(\varepsilon_{V}+\mu_{V}\right)\left(b \beta_{V} \varepsilon_{H} \mu_{H}+\left(\gamma_{H}+\mu_{H}\right)\left(\varepsilon_{H}+\mu_{H}\right) \mu_{V}\right)\right] \\
W_{1}= & \left(-\gamma_{H}-\mu_{H}\right)\left(-\varepsilon_{H}-\mu_{H}\right) \\
W_{2}= & \left(-\varepsilon_{V}-\mu_{V}\right) \mu_{V} \\
W_{3}= & \left(\gamma_{H}+\varepsilon_{H}+2 \mu_{H}\right) \\
W_{4}= & \left(\varepsilon_{V}+2 \mu_{V}\right) \\
Y_{1}= & \left(b N_{V} \beta_{H} \varepsilon_{V}+N_{H} \mu_{H}\left(\varepsilon_{V}+\mu_{V}\right)\right) \\
& b N_{H}^{2} N_{V}^{2} \beta_{H}^{2} \beta_{V}^{2} \varepsilon_{H}^{2} \varepsilon_{V}^{2}\left(\varepsilon_{V}+\mu_{V}\right)^{3} \\
Y_{2}= & \left.\left(b \beta_{V} \varepsilon_{H} \mu_{H}+\left(\gamma_{H}+\mu_{H}\right)\left(\varepsilon_{H}+\mu_{H}\right) \mu_{V}\right)\right), \\
Y_{3}= & N_{H}^{2} N_{V} \beta_{H} \beta_{V}^{2} \varepsilon_{H}^{2} \varepsilon_{V}
\end{aligned}
$$

These coefficients of this new characteristic equation will also satisfy the Routh-Hurwitz criteria, Equation (32) - (34) for the coefficients defined above (Fig. 6) and so the eigenvalues by the characteristic equation above will all have negative imaginary parts and the endemic disease equilibrium point will be stable.

\section{Numerical Results}

The numerical analysis in this study considers the transmission of dengue disease in models where the values of the parameter values are listed in Table 1, which gives different values for three sets of parameters which leads to the three cases we are looking at. Case 1 are the values when vertical transmission occurs and the equilibrium state is the endemic state. Case 2 are the values when there is no vertical transmission is possible but the equilibrium state will be the disease free state. Finally, case 3 are the values when there is no vertical transmission but the equilibrium state will be the endemic state.

The trajectories of the numerical solutions case 1, case 2 and case 3 projected onto $S_{H}, E_{H}, I_{H}, E_{V}$ and $I_{V}$ are shown in the Fig. 7-9 respectively. The trajectory of the numerical solutions case 1 , case 2 and case 3 projected onto $\left(S_{H}, E_{H}\right),\left(S_{H}, I_{H}\right),\left(S_{H}, E_{V}\right),\left(S_{H}, I_{V}\right),\left(E_{H}\right.$, $\left.E_{V}\right)$ and $\left(I_{H}, I_{V}\right)$ are shown in the Fig. 10-12 respectively. 
Table 1: Parameter are used involved in the transmission of dengue disease

\begin{tabular}{llll}
\hline Parameter & Case 1 & Case 2 & Case 3 \\
\hline$\mu_{\mathrm{H}}$ & $1 /(70 * 365)$ & $1 /(70 * 365)$ & $1 /(70 * 365)$ \\
$\mathrm{N}_{\mathrm{H}}$ & 92,000 & 92,000 & 92,000 \\
$b$ & $1 / 5$ & $1 / 5$ & $1 / 5$ \\
$A$ & 5,000 & 5,000 & 5,000 \\
$\mu_{\mathrm{V}}$ & $1 / 24$ & $1 / 24$ & $1 / 24$ \\
$M$ & 400 & 0 & 0 \\
$\gamma_{\mathrm{H}}$ & 0.1428 & 0.01428 & 0.01428 \\
$\beta_{\mathrm{H}}$ & 0.95 & 0.65 & 0.65 \\
$\beta_{\mathrm{V}}$ & 0.75 & 0.65 & 0.65 \\
$\varepsilon_{\mathrm{V}}$ & 0.1428 & 0.01428 & 0.1428 \\
$\varepsilon_{\mathrm{H}}$ & 0.1667 & 0.01667 & 0.1667 \\
\hline
\end{tabular}

The trajectory of the numerical solutions case 1 , case 2 and case 3 projected onto $\left(S_{H}, E_{H}, I_{H}\right),\left(S_{H}, E_{H}, E_{V}\right),\left(S_{H}\right.$, $\left.E_{H}, I_{V}\right),\left(S_{H}, E_{V}, I_{V}\right),\left(E_{H}, E_{V}, I_{V}\right)$ and $\left(I_{H}, E_{V}, I_{V}\right)$ are shown in the Fig. 13-15 respectively.

\section{Discussion}

In this study, the dynamic transmission of dengue disease using SEIR mathematical models which focus on the transmission of the virus in the mosquito by its being bitten by an infected human or by vertical transmission mode, i.e., through sexual contact with a male mosquito is studied. It is shown that the presence of vertical transmission insures that the endemic equilibrium state is the only possible outcome. In the absence of vertical transmission, the model leads to two possible outcomes, a disease free equilibrium state and an endemic equilibrium state which depend on whether $R_{0}<1$ or $R_{0}>1$. The Routh-Hurwitz criteria for the coefficients of the characteristics equations for

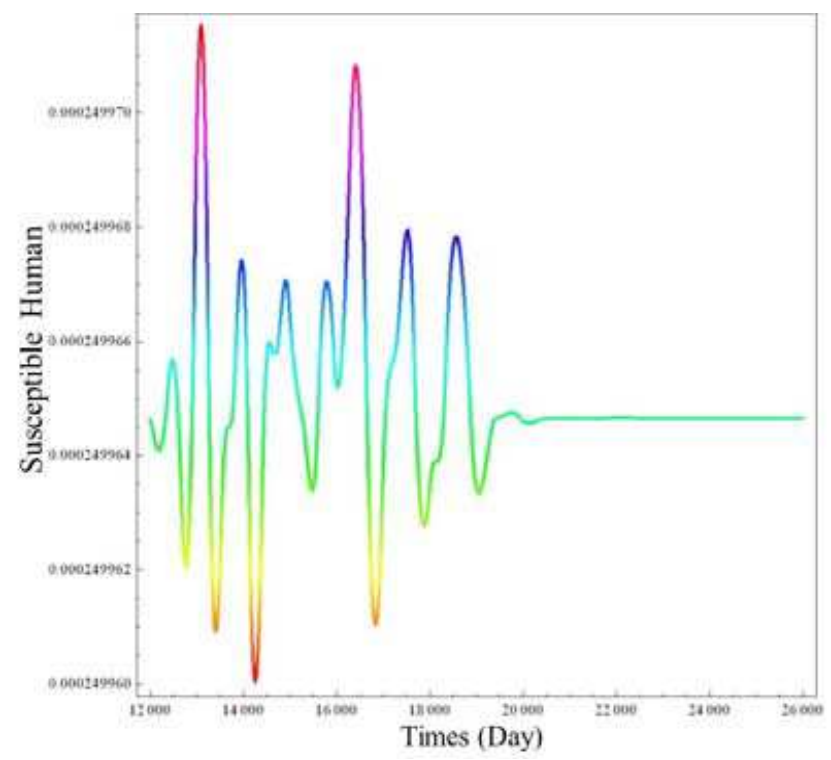

the system are used to determine whether all the eigenvalues have negative imaginary parts.

When there is vertical transmission of the virus in the mosquito and the values of the parameters are such that $R_{0}>1$, the only equilibrium state is the endemic equilibrium point, $E_{l}$ and it is local asymptotically stable as can be seen from Fig. 4 which shows the values of the parameters satisfy the RouthHurwitz criteria. The time trajectories of $S_{H}, E_{H}, I_{H}, E_{V}$ and $I_{V}$ are plotted on Fig. 7. The trajectories of the numerical solutions are plotted on the 2D $\left(S_{H}, E_{H}\right)$, $\left(S_{H}, I_{H}\right),\left(S_{H}, E_{V}\right),\left(S_{H}, I_{V}\right),\left(E_{H}, E_{V}\right)$ and $\left(I_{H}, I_{V}\right)$ planes and in the 3D $\left(S_{H}, E_{H}, I_{H}\right),\left(S_{H}, E_{H}, E_{V}\right),\left(S_{H}, E_{H}, I_{V}\right)$, $\left(S_{H}, E_{V}, I_{V}\right),\left(E_{H}, E_{V}, I_{V}\right)$ and $\left(I_{H}, E_{V}, I_{V}\right)$ spaces seen in Fig. 10 and 13 respectively.

In the absence of vertical transmission, the disease free equilibrium point, $E_{0}$, will be local asymptotically stable when $R_{0}<1$. The range of values of the parameters for which the coefficients of the characteristic equation for eigenvalues satisfy the Routh-Hurwitz criteria for the disease free state to be local asymptotical stable are shown in Fig. 5.

Picking the values (the ones listed for case 2 in Table 1 and given in figure caption), the time dependences of $S_{H}, E_{H}, I_{H}, E_{V}$ and $I_{V}$, are plotted in Fig. 8. The trajectories of the numerical solutions are plotted in the $2 \mathrm{D}\left(S_{H}, E_{H}\right),\left(S_{H}, I_{H}\right),\left(S_{H}, E_{V}\right),\left(S_{H}, I_{V}\right)$, $\left(E_{H}, E_{V}\right)$ and $\left(I_{H}, I_{V}\right)$ and the $3 \mathrm{D}\left(S_{H}, E_{H}, I_{H}\right),\left(S_{H}, E_{H}\right.$, $\left.E_{V}\right),\left(S_{H}, E_{H}, I_{V}\right),\left(S_{H}, E_{V}, I_{V}\right),\left(E_{H}, E_{V}, I_{V}\right)$ and $\left(I_{H}, E_{V}\right.$, $\left.I_{V}\right)$ space in Fig. 11 and 14 respectively. The endemic equilibrium point, $E_{2}$, is local asymptotically stable for $R_{0}>1$. The behaviors of the populations for this case (case 3) are shown in Fig. 6, 9 and 15.

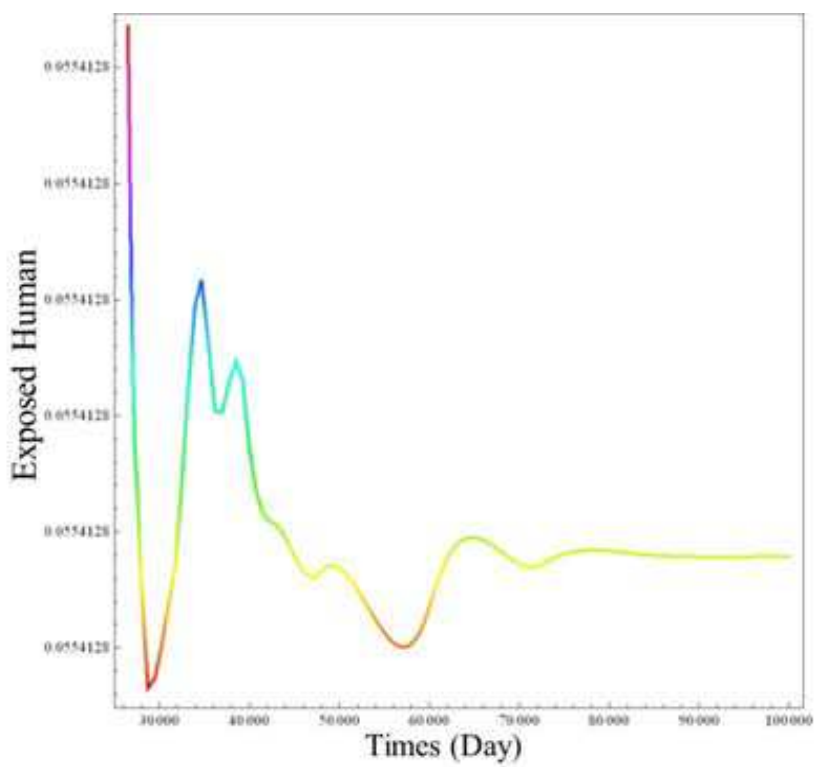


Pratchaya Chanprasopchai et al. / American Journal of Applied Sciences 2017, 14 (12): 1123.1145
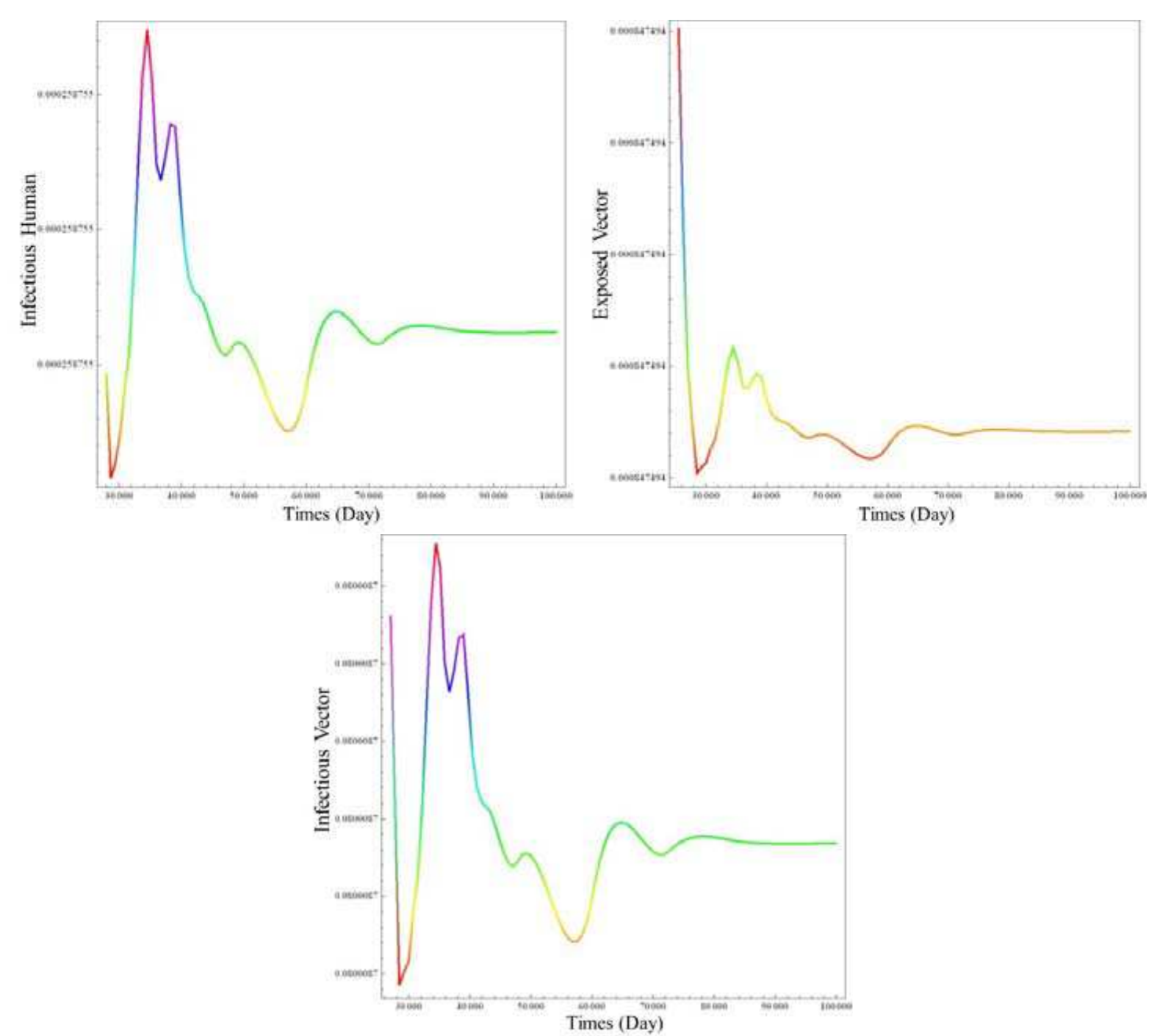

Fig. 7: The time trajectories of the numerical solutions of the model when vertical transmission occurs for $S_{H}, E_{H}, I_{H}, E_{V}$ and $I_{V}$ are shown
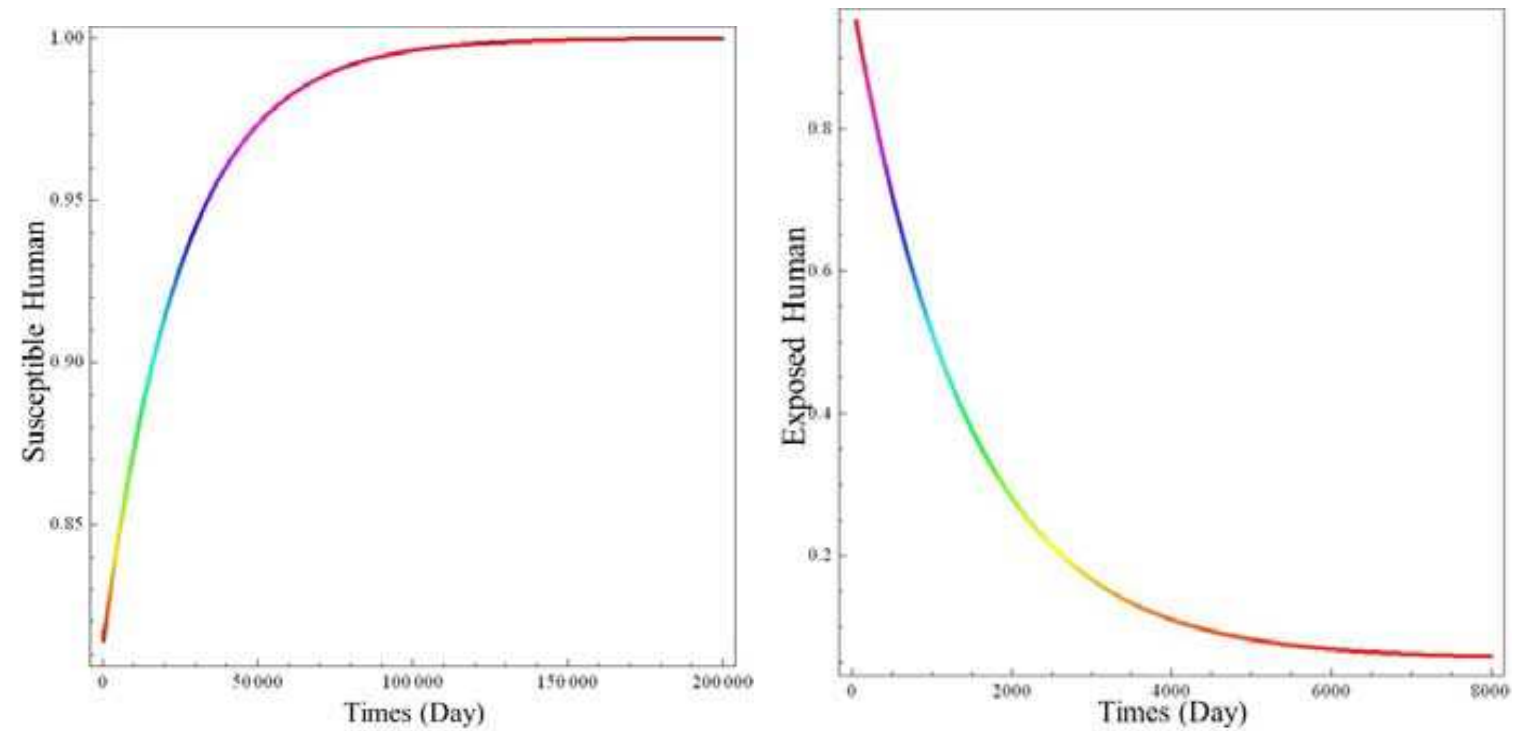

1135 

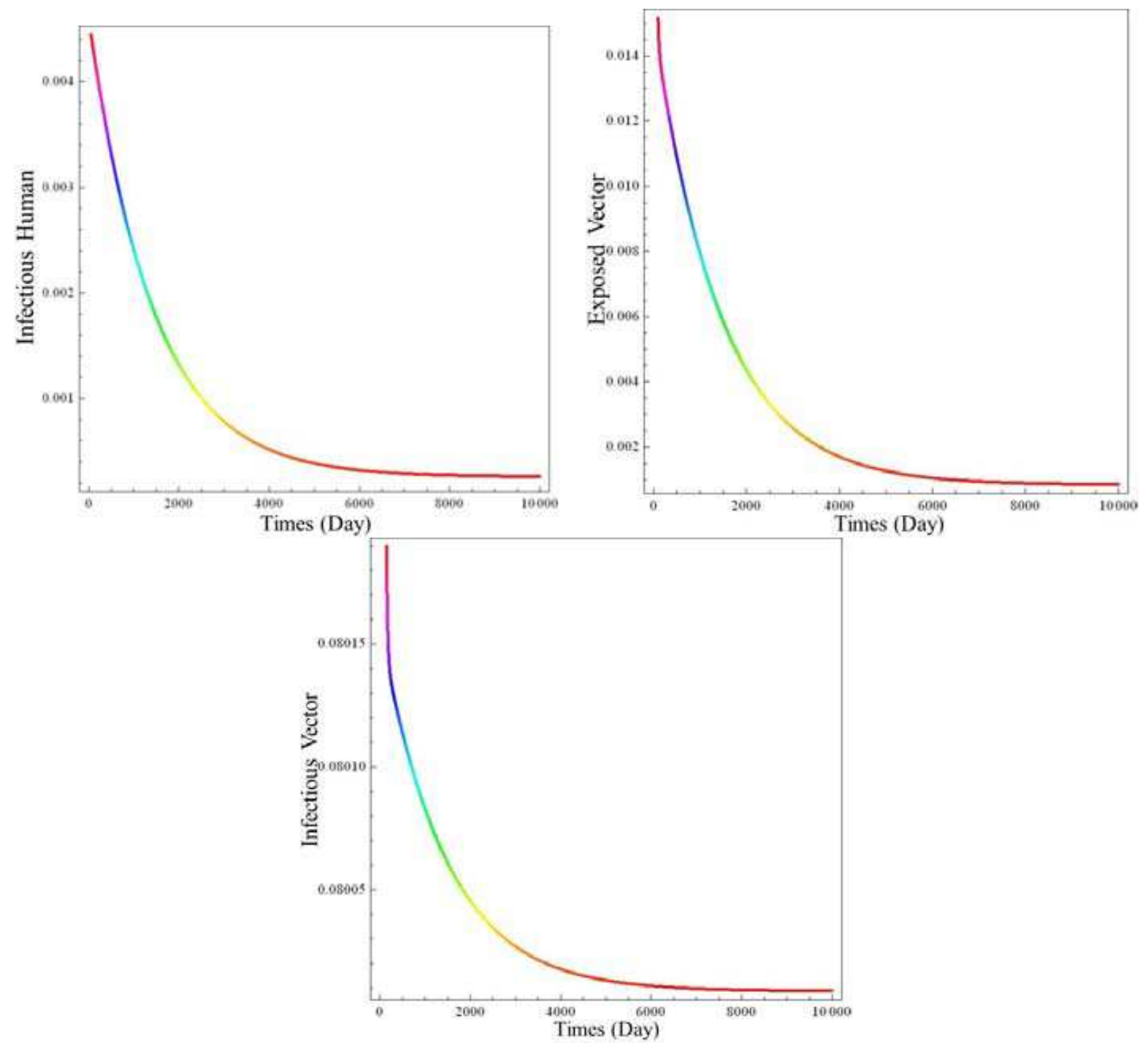

Fig. 8: The time trajectories of the numerical solutions of the model when there is no vertical transmission of the virus of $S_{H}, E_{H}, I_{H}, E_{V}$ and $I_{V}$ lead to the equilibrium state being the disease free state
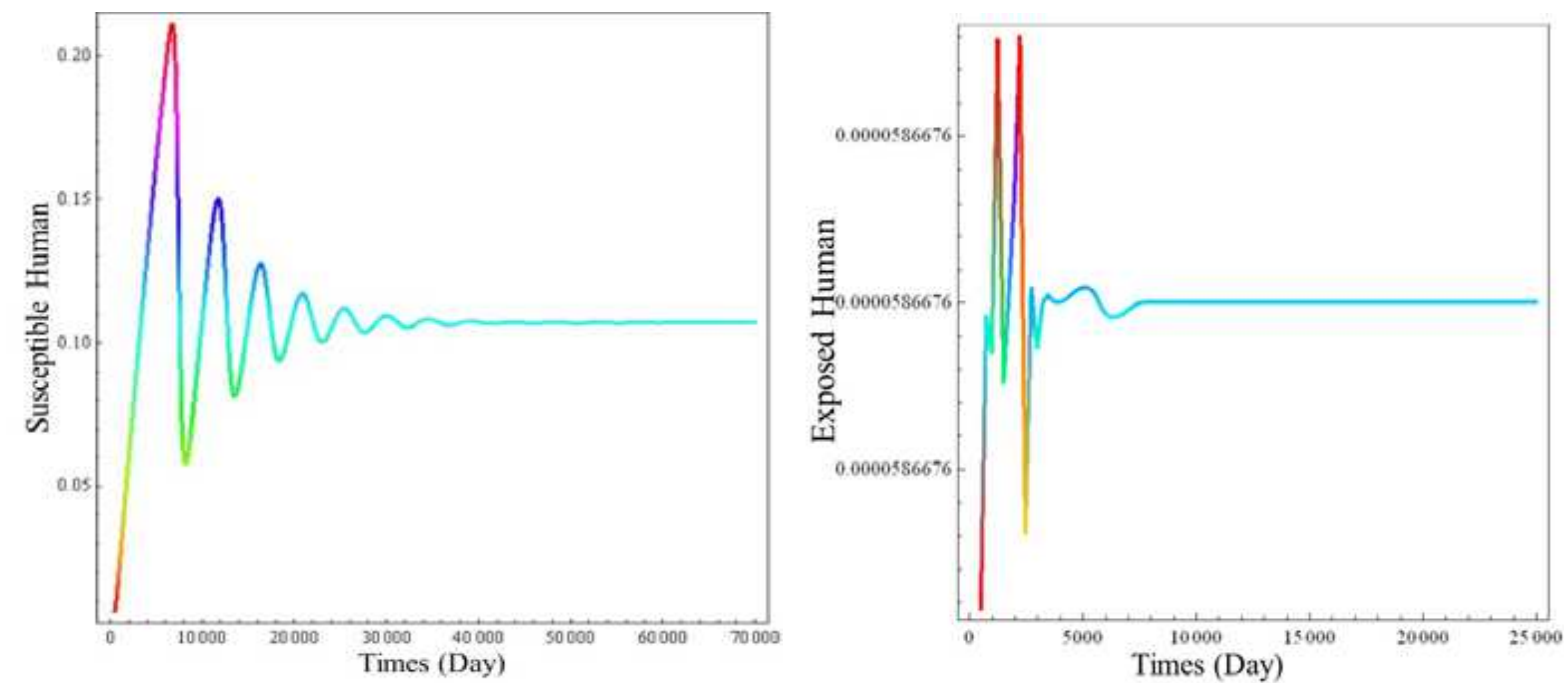


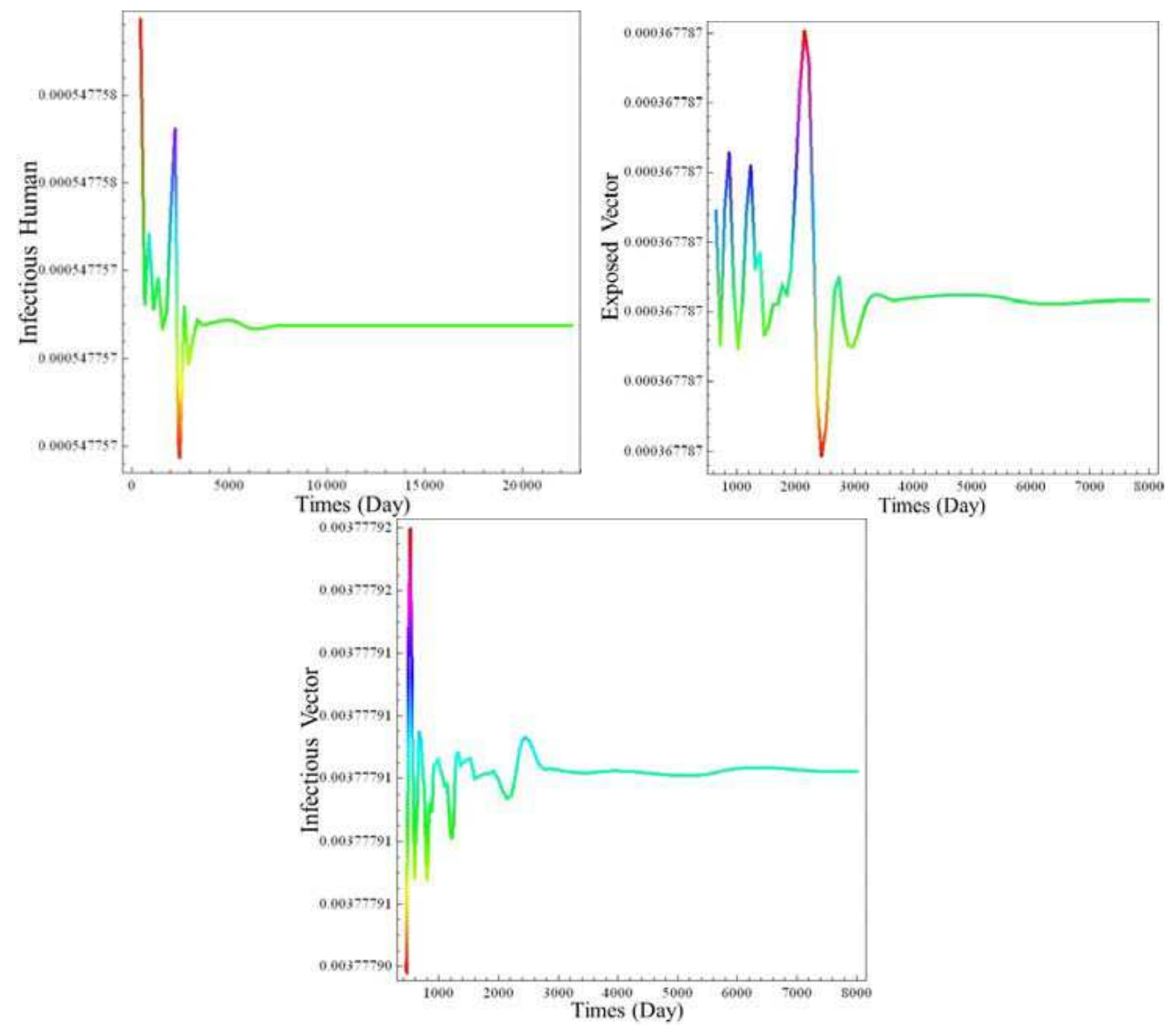

Fig. 9: The time trajectories of the numerical solutions of the model when there is no vertical transmission of the virus of $S_{H}, E_{H}, I_{H}$, $E_{V}$ and $I_{V}$ lead to the equilibrium state being the endemic disease state
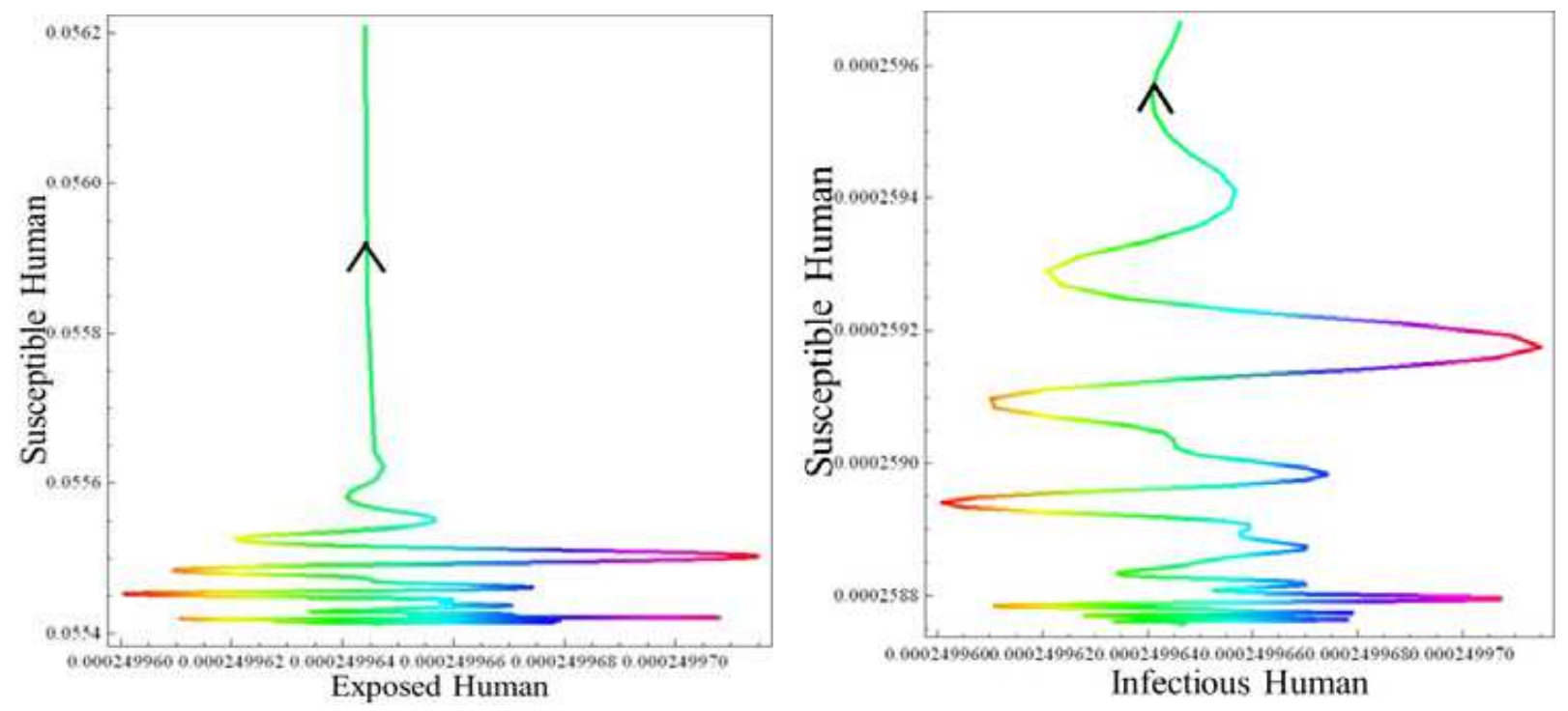

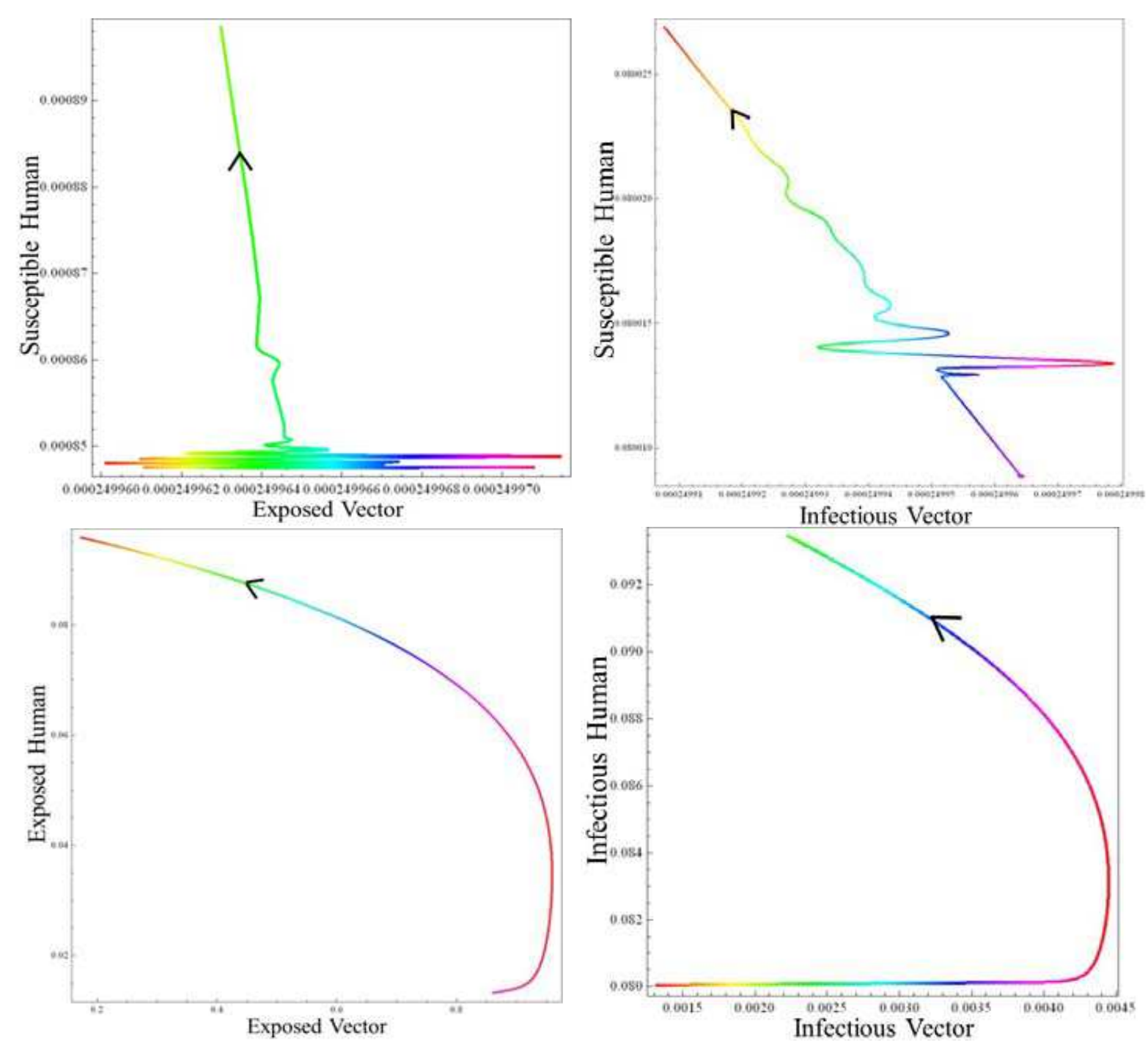

Fig. 10: The trajectories of the numerical solutions projected onto the $2 \mathrm{D}\left(S_{H}, E_{H}\right),\left(S_{H}, I_{H}\right),\left(S_{H}, E_{V}\right),\left(S_{H}, I_{V}\right),\left(E_{H}, E_{V}\right)$ and $\left(I_{H}, I_{V}\right)$ planes when vertical transmission occurs
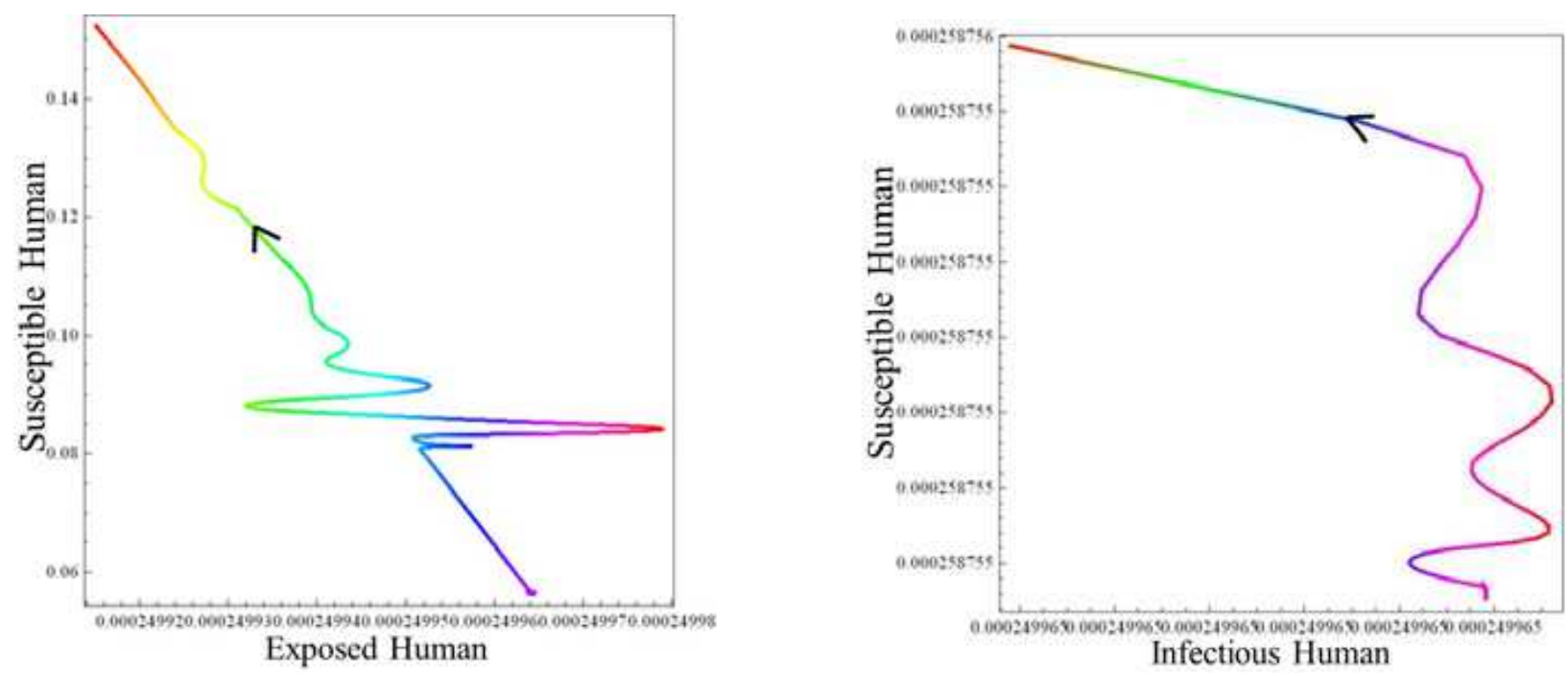

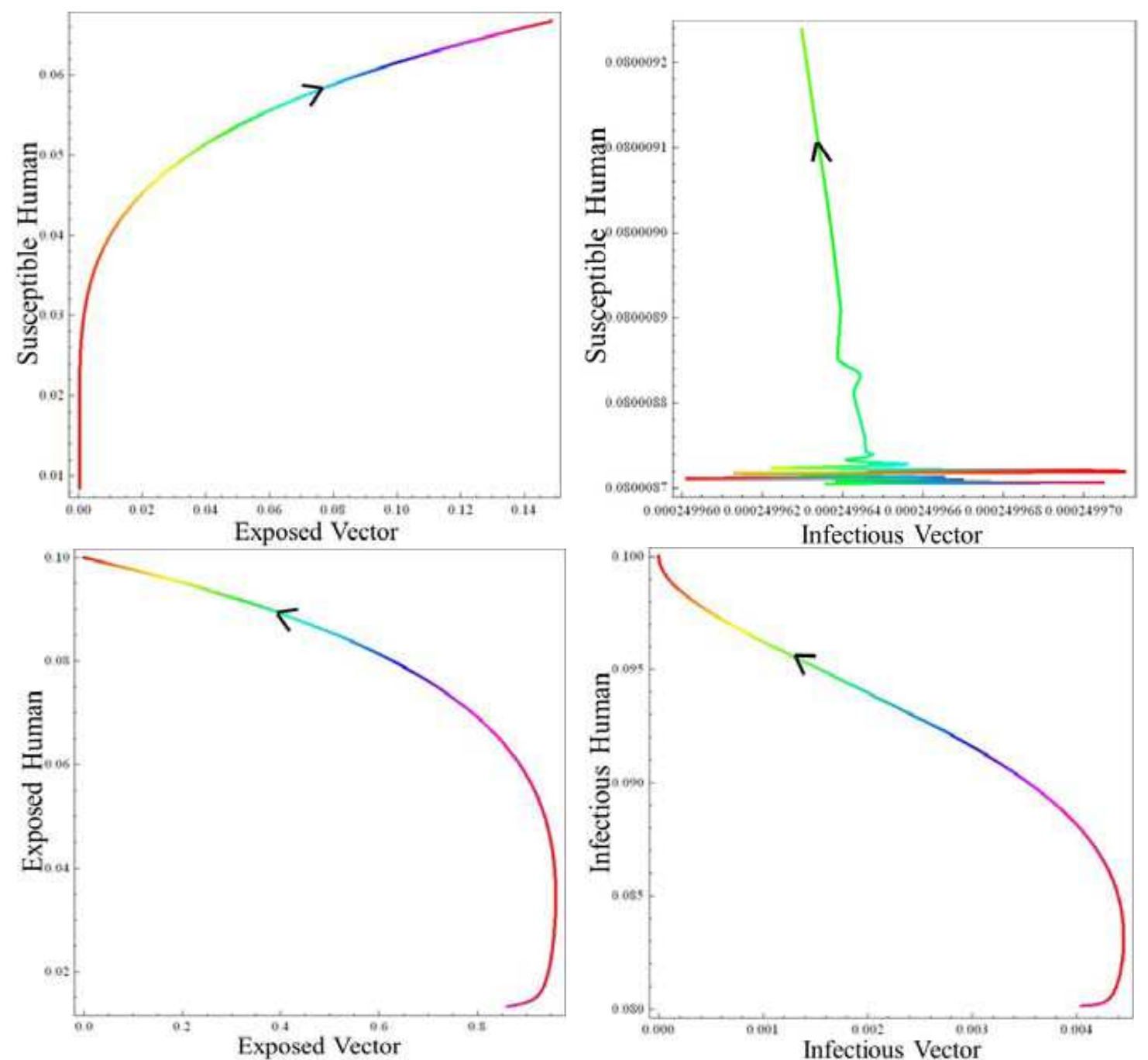

Fig. 11: The trajectories of the numerical solutions projected onto the $2 \mathrm{D}\left(S_{H}, E_{H}\right),\left(S_{H}, I_{H}\right),\left(S_{H}, E_{V}\right),\left(S_{H}, I_{V}\right),\left(E_{H}, E_{V}\right)$ and $\left(I_{H}, I_{V}\right)$ planes when there is no vertical transmission and equilibrium state is the disease free state
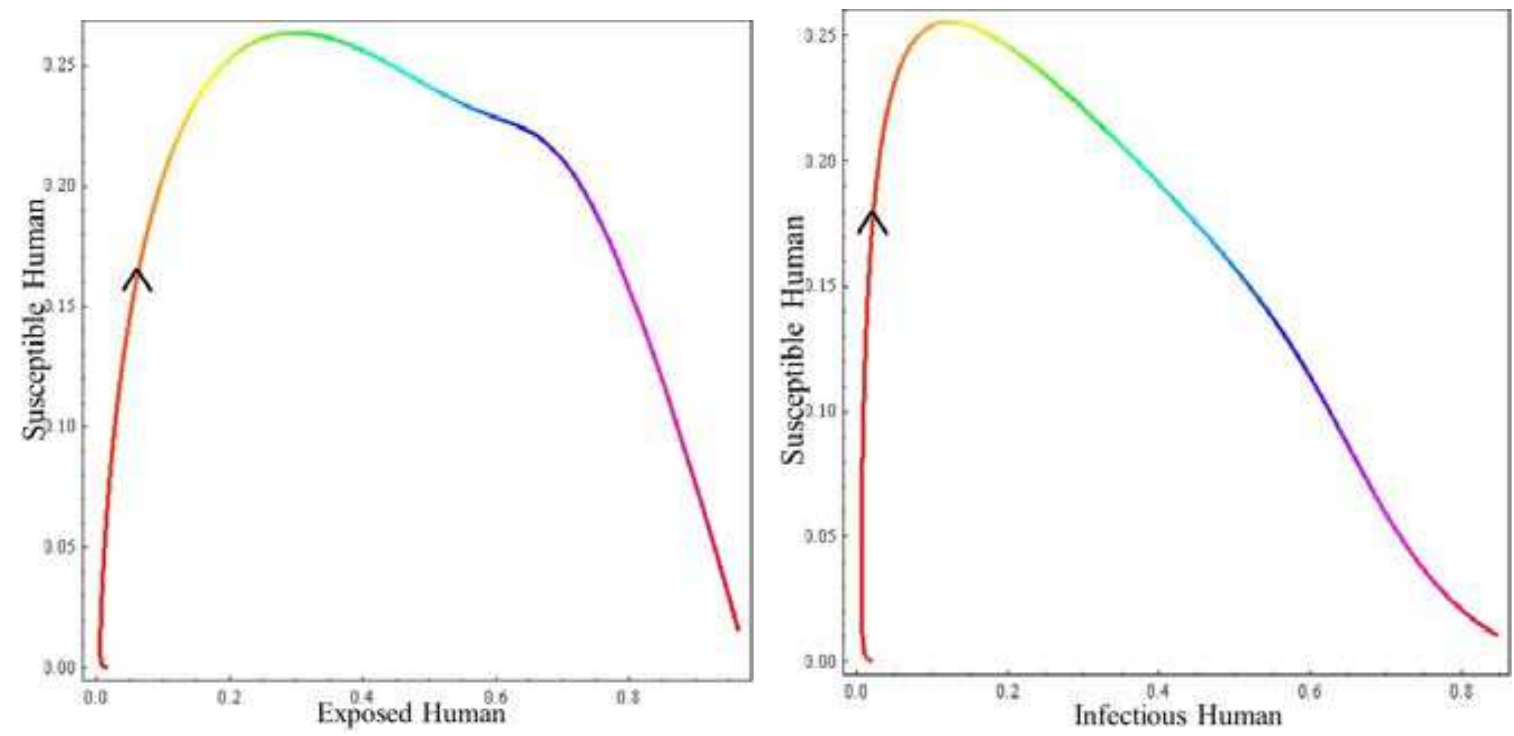

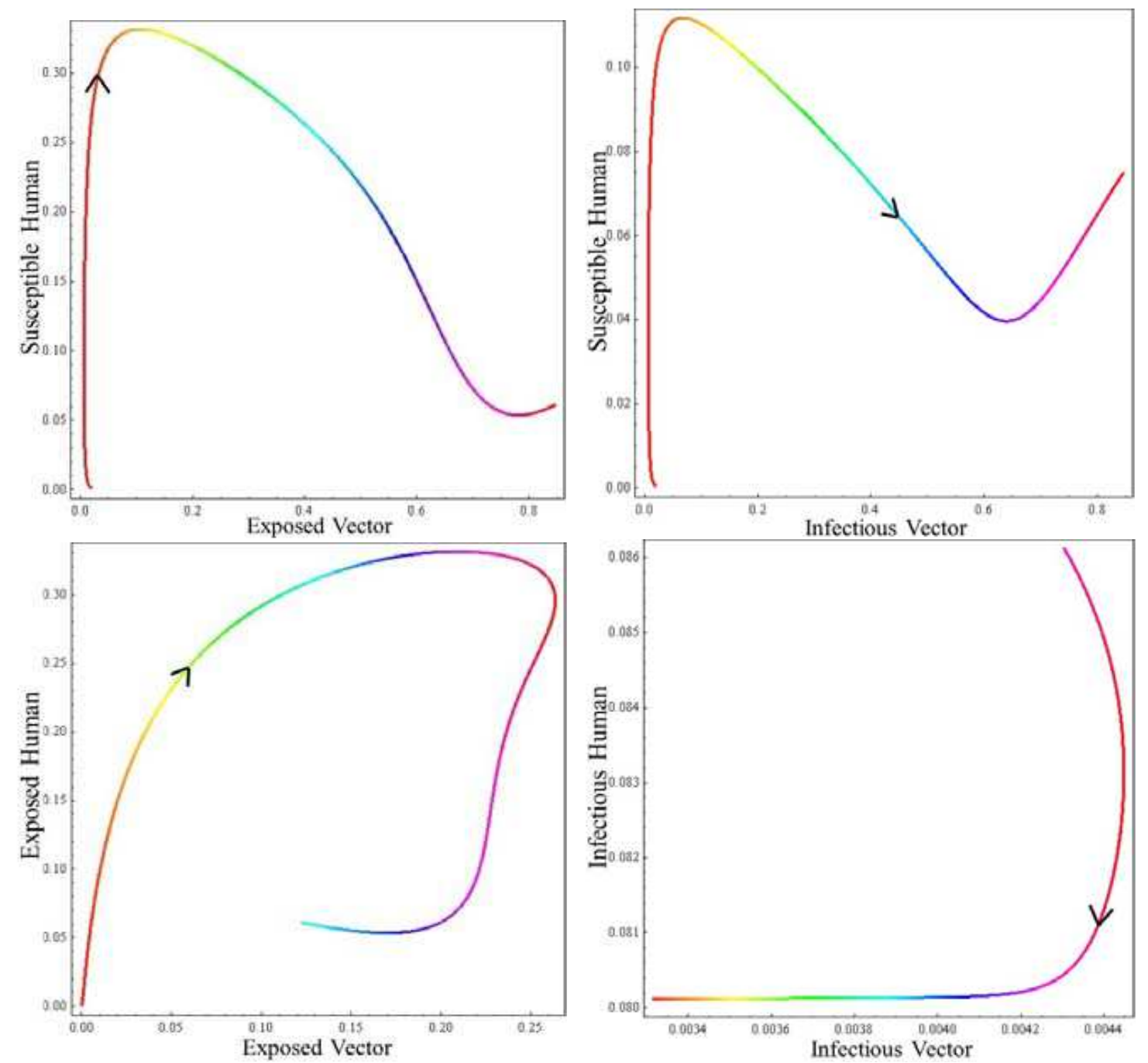

Fig. 12: The trajectories of the numerical solutions projected onto the $2 \mathrm{D}\left(S_{H}, E_{H}\right),\left(S_{H}, I_{H}\right),\left(S_{H}, E_{V}\right),\left(S_{H}, I_{V}\right),\left(E_{H}, E_{V}\right)$ and $\left(I_{H}, I_{V}\right)$ planes when there is no vertical transmission and equilibrium state is the endemic state
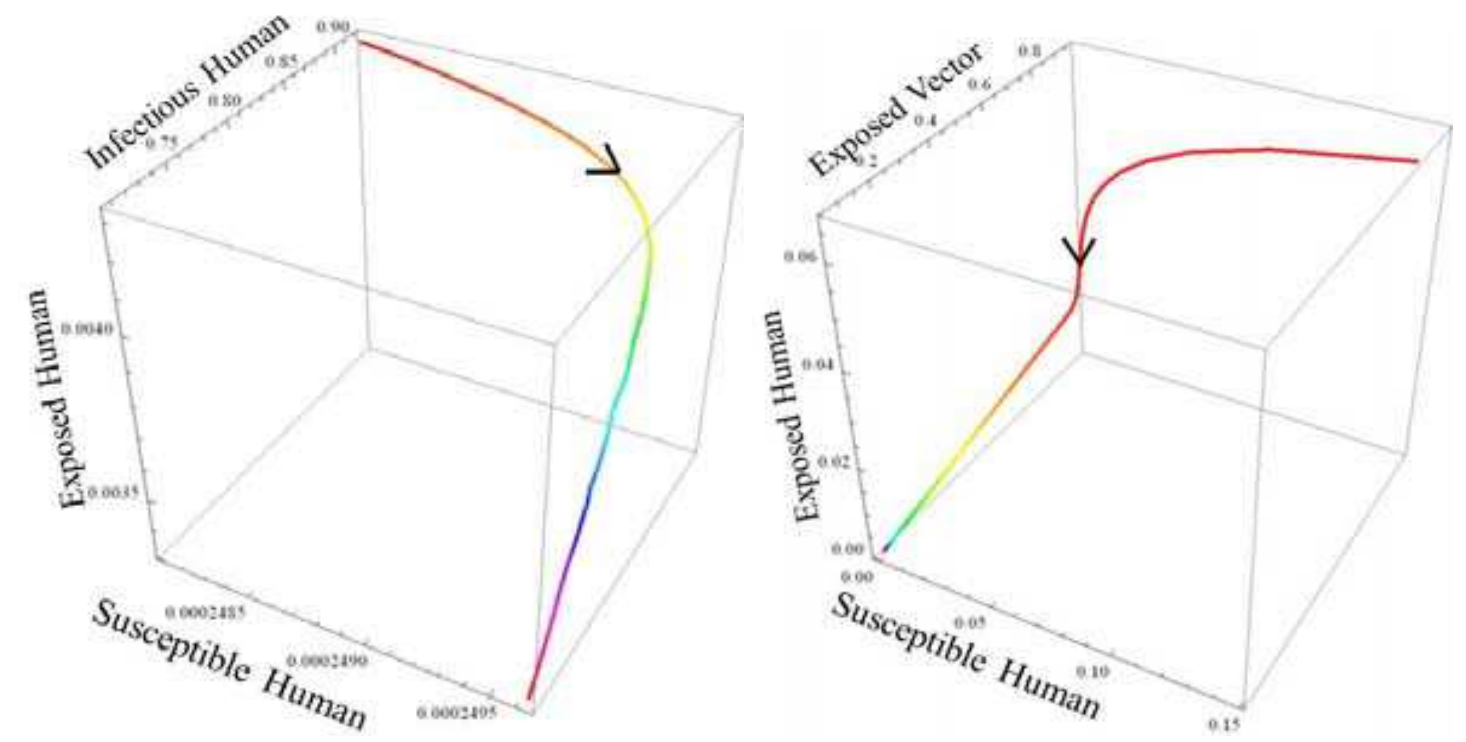

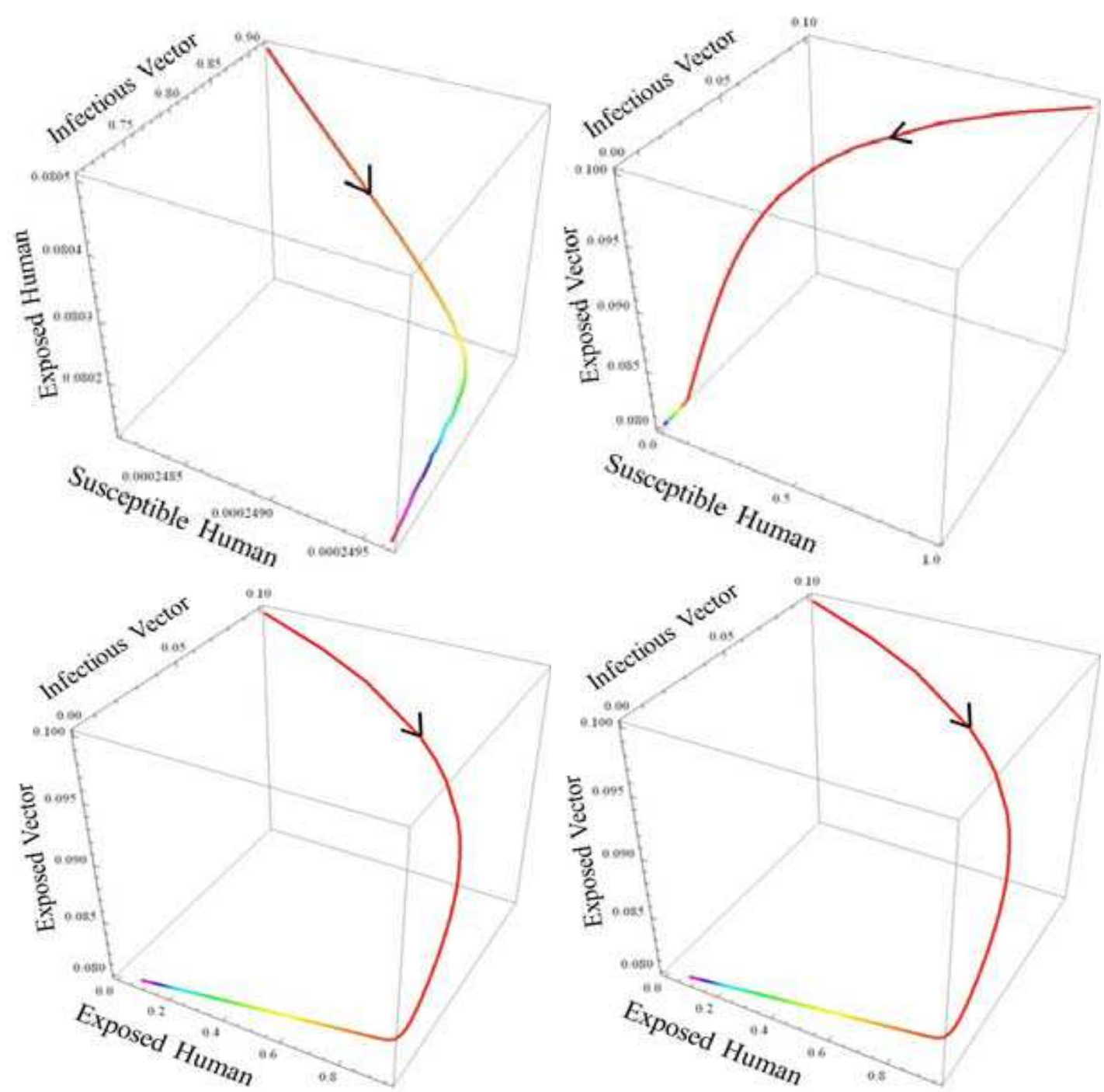

Fig. 13: The trajectories of the numerical solutions of the model when vertical transmission occurs into the $3 \mathrm{D}\left(S_{H}, E_{H}, I_{H}\right),\left(S_{H}, E_{H}\right.$, $\left.E_{V}\right),\left(S_{H}, E_{H}, I_{V}\right),\left(S_{H}, E_{V}, I_{V}\right),\left(E_{H}, E_{V}, I_{V}\right)$ and $\left(I_{H}, E_{V}, I_{V}\right)$ spaces
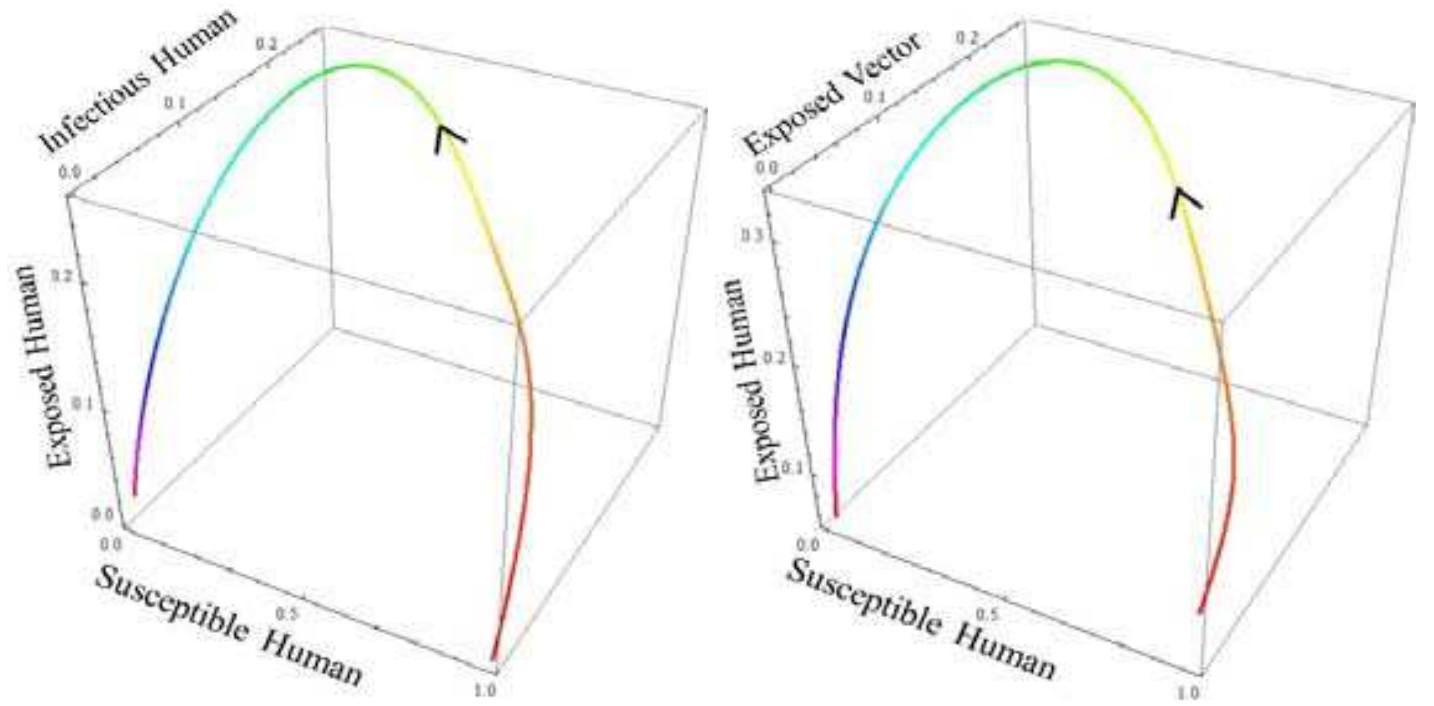

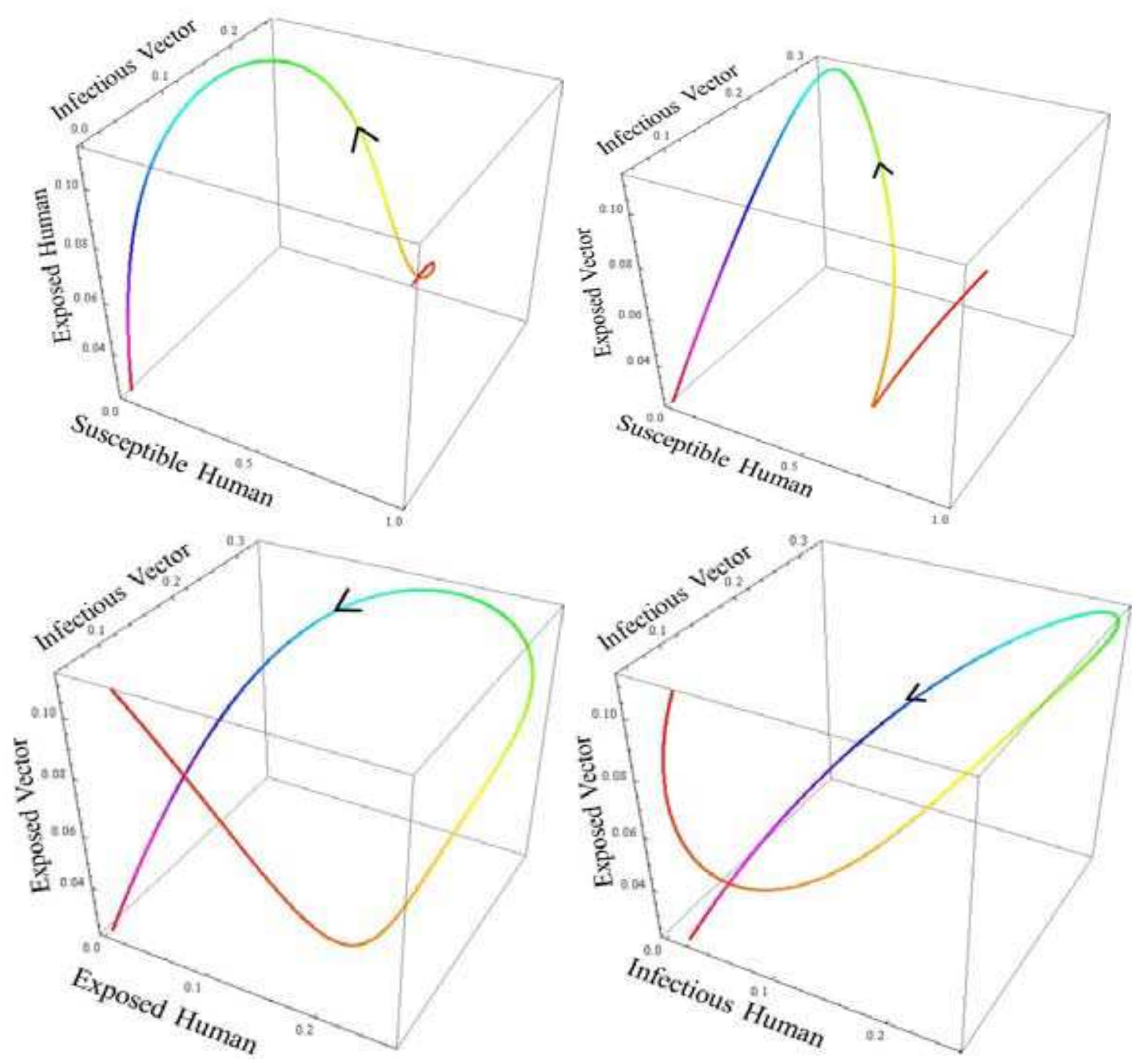

Fig. 14: The trajectories of the numerical solutions into the $3 \mathrm{D}\left(S_{H}, E_{H}, I_{H}\right),\left(S_{H}, E_{H}, E_{V}\right),\left(S_{H}, E_{H}, I_{V}\right),\left(S_{H}, E_{V}, I_{V}\right),\left(E_{H}, E_{V}, I_{V}\right)$ and $\left(I_{H}, E_{V}, I_{V}\right)$ spaces when there is no vertical transmission and the values of the parameters are such that the equilibrium state is the disease free state
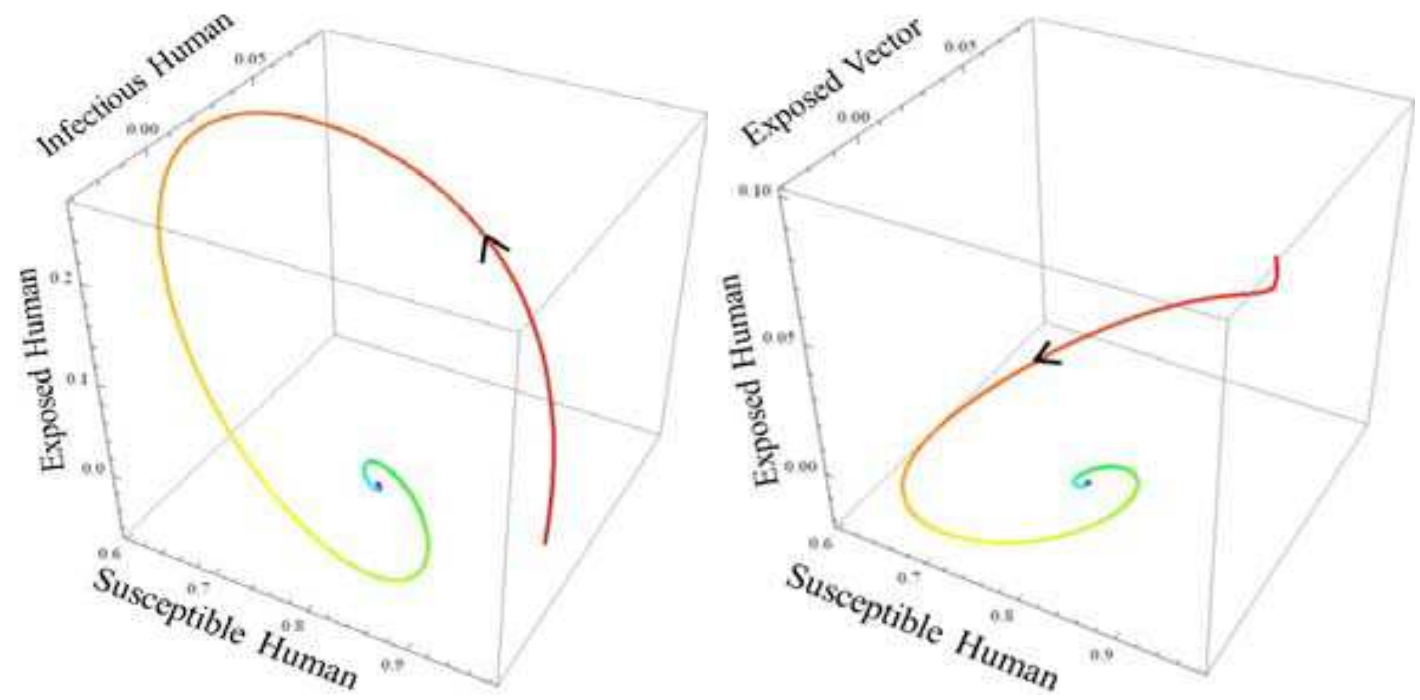

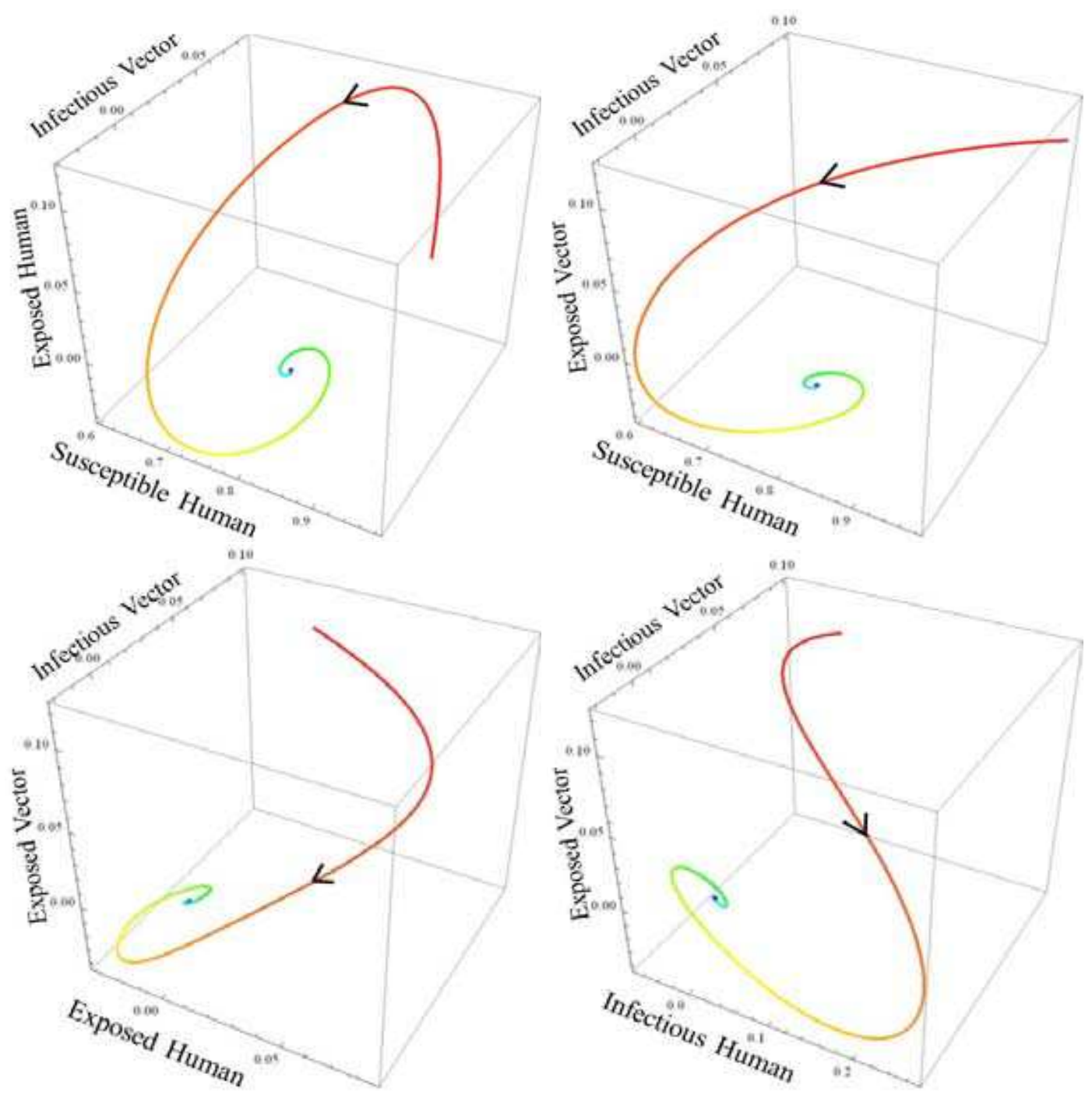

Fig. 15: The trajectories of the numerical solutions into the 3D $\left(S_{H}, E_{H}, I_{H}\right),\left(S_{H}, E_{H}, E_{V}\right),\left(S_{H}, E_{H}, I_{V}\right),\left(S_{H}, E_{V}, I_{V}\right),\left(E_{H}, E_{V}, I_{V}\right)$ and $\left(I_{H}, E_{V}, I_{V}\right)$ spaces when there is no vertical transmission and the values of the parameters are such that the equilibrium state is the endemic disease state

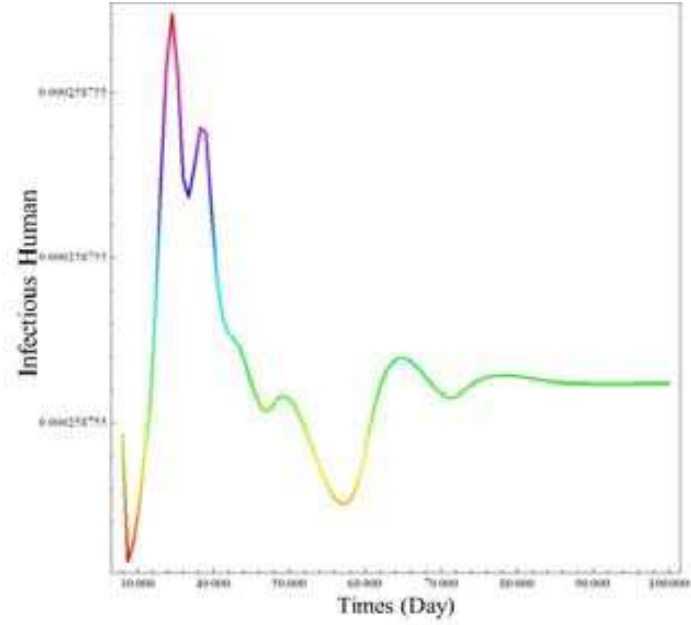

In the presence of vertical transmission of the virus

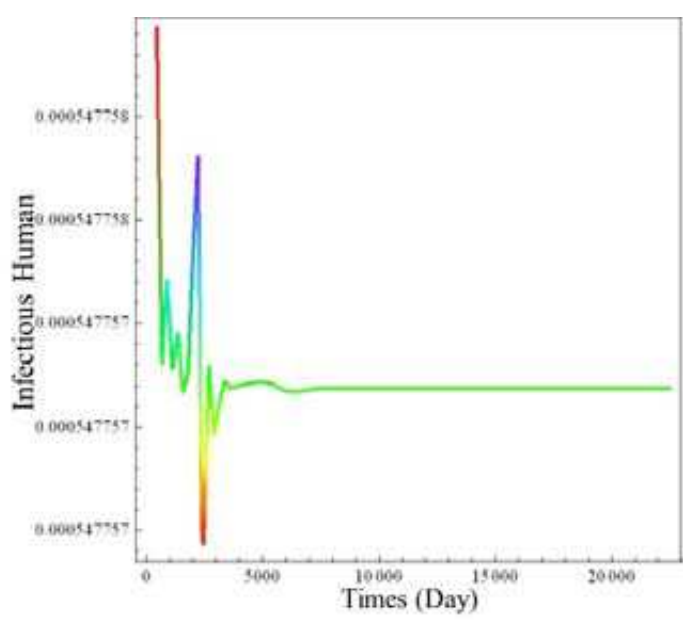

(a)

In the absence of vertical transmission of the virus 

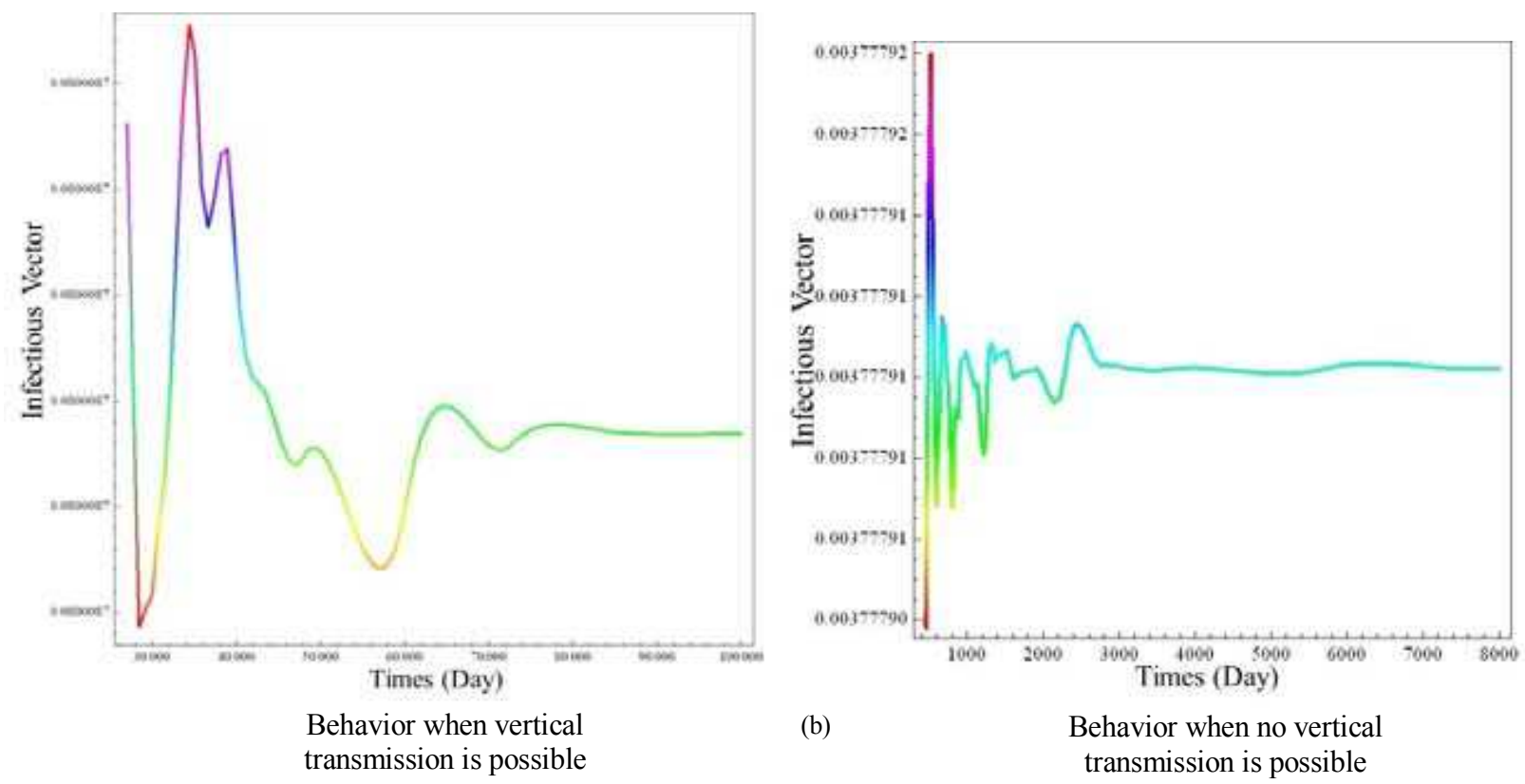

Fig. 16: (a) The time series comparison of dengue disease with and without the effect of vertical transmission for infectious human, $\mathrm{IH}$ (b) the time series comparison of dengue disease with and without the effect of vector born infection projected onto infectious vector, $I_{V}$

\section{Conclusion}

To see the influence of vertical transmission in the mosquitoes on the human and mosquito populations, we have plotted on Fig. 16(a) and 16(b) the time dependence of the infectious humans and mosquitoes in the presence or absence of vertical transmission of the virus in the mosquitoes of $I_{H}$ and $I_{V}$. In both cases, the equilibrium state was the endemic state. We see that the equilibrium states were reached slower when vertical transmission of the virus in the mosquito occurs.

\section{Acknowledgement}

This study is supported by King Mongkut's Institute of Technology Ladkrabang and National Research Council of Thailand. I Ming Tang acknowledge the financial support provided by King Mongkut's University of Technology, Thonburi through the KMUTT 55th Anniversary Commemorative Fund.

\section{Author's Contributions}

Pratchaya Chanprasopchai: Make considerable contributions to conception and design and acquisition of data, analysis and interpretation of data.

I Ming Tang: Give the ideal of the problem and give final approval of the version to be submitted and any revised version.

Puntani Pongsumpun: Make considerable contributions to conception and design or acquisition of data and/or Analysis and interpretation of data and contribute in drafting the article or reviewing it critically for significant intellectual content.

\section{Conflict of Interests}

The authors declare that there is no conflict of interest regarding this research.

\section{References}

Aldila, D., T. Götz and E. Soewonoa, 2013. An optimal control problem arising from a dengue disease transmission model. Math. Biosci., 242: 9-16. DOI: $10.1016 /$ j.mbs.2012.11.014

Bakach, I. and J. Braselton, 2015. A survey of mathematical models of dengue Fever. J. Comput. Sci. Syst. Biol., 8: 255-267. DOI: $10.4172 /$ jcsb. 1000198

Bureau of Epidemiology, 2015. Ministry of Public Health. 1st Edn., Annual Report, Bangkok, Thailand.

D’Ortenzio, E., S. Matheron, Y. Yazdanpanah, X. de Lamballerie and B. Hubert et al., 2016. Evidence of sexual transmission of Zika Virus. N. Eng. J. Med. 347: 2195-2198. DOI: 10.1056/NEJMc1604449 DOI: $10.1080 / 00207160.2011 .554540$

Erickson, R.A., S.M. Presleyab, L.J.S. Allenbc, K.R. Long and S.B. Cox, 2010. A dengue model with a dynamic Aedes albopictus vector population. Ecol. Model., 221: 2899-2908. DOI: 10.1016/j.ecolmodel.2010.08.036

Esteva, L. and C. Vargas, 2000. Influence of vertical and mechanical transmission of dengue fever. Math. Biosci. 165: 15-64. 
Esteva, L. and C. Vargus, 1999. Analysis of a dengue disease transmission model. Math. Biosci., 150: 131-151.

Grunnil, M. and M. Boots, 2016. How important is vertical transmission of dengue virus by mosquitoes (Diptera Culicidae). J. Med. Entomol. 53: 1-19.

Hiroshi, N., 2006. Mathematical and statistical analyses of the spread of dengue. Math. Approaches Dengue, Dengue Bull., 30: 51-67.

Lequime, S., R.E. Paul and L. Lambrechts, 2016. Determinants of arbovirus vertical transmission in mosquitoes. PLoS Pathog.

Naowarat, S., T. Korkiatsakul and I.M. Tang, 2011. Dynamical model for determining human susceptibility to dengue fever. Am. J. Applied Sci., 8: 1101-1106.

Pongsumpun, P., 2006a. Dengue disease model with the effect of extrinsic incubation period. Proceedings of the WSEAS International Conference on Mathematical Biology and Ecology, (MBE' 06), Miami, Florida, USA: pp: 43-48.

Pongsumpun, P., 2006b. Transmission model for dengue disease with and without the effect of extrinsic incubation period. KMITL Sci. Tech. J., 6: 74-82.

Pongsumpun, P., 2007. Effect of incubation period of virus for the mathematical model of dengue disease. Proceedings of the 3rd WSEAS/IASME International Conference on Dynamical Systems and Control, (DSC' 07), Arachon, France, pp: 182-187.
Pongsumpun, P., 2011. Seasonal transmission model of dengue virus infection in Thailand. J. Basic Applies Scientific Res., 1: 1372-1379.

drigues, H.S., M. Teresa, T. Monteiro, F.D.M. Torres and A. Zinober, 2011. Dengue disease, basic reproduction number and control. Int. J. Comput. Math., 3: 334-346.

Thenmozhi, V., J.G. Hiriyan, S.C. Tewari, S.P. Philip and R. Paramasivan et al., 2007. Natural vertical transmission of dengue virus in Aedes albopictus (Ditera: Culicidae) in Keralia, a Southern India state. Jpn. J. Infect. Dis., 60: 245-249.

WHO, 2011. Dengue, guidelines for diagnosis, treatment, prevention and control. Geneva, Switzerland World Health Organization.

WHO, 2012. Global strategy for dengue prevention and control 2012-2020. Geneva, Switzerland World Health Organization.

Yin, X., X. Zhong and S. Pan, 2010. Vertical transmission of dengue infection: The first putative case reported in China. Rev. Inst. Med. Trop. Sao Paulo, 28: 58-90. DOI: $10.1590 / \mathrm{S} 1678-9946201658090$ 